\title{
Some Specific CASL Requirements for Advanced Multiphase Flow Simulation of Light Water Reactors
}

R. A. Berry

November 2010

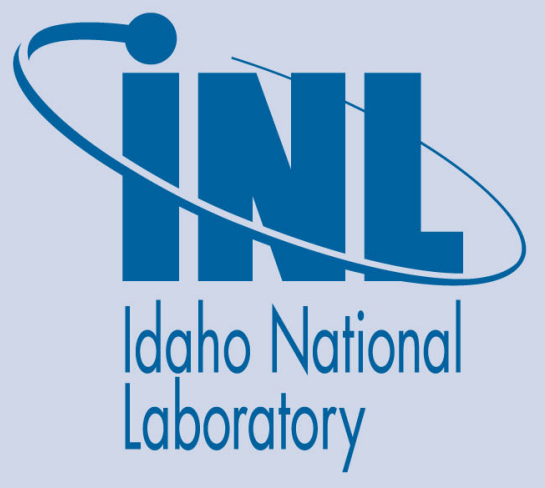

The INL is a U.S. Department of Energy National Laboratory operated by Battelle Energy Alliance 


\title{
Some Specific CASL Requirements for Advanced Multiphase Flow Simulation of Light Water Reactors
}

\author{
R. A. Berry
}

November 2010

\section{Idaho National Laboratory \\ Idaho Falls, Idaho 83415}

http://www.inl.gov

Prepared for the

U.S. Department of Energy

Office of Nuclear Energy

Under DOE Idaho Operations Office

Contract DE-AC07-05ID14517 


\section{DISCLAIMER}

This information was prepared as an account of work sponsored by an agency of the U.S. Government. Neither the U.S. Government nor any agency thereof, nor any of their employees, makes any warranty, expressed or implied, or assumes any legal responsibility for the accuracy, completeness, or usefulness, of any information, apparatus, product, or process disclosed, or represents that its use would not infringe privately owned rights. References herein to any specific commercial product, process, or service by trade name, trade mark, manufacturer, or otherwise, does not necessarily constitute or imply its endorsement, recommendation, or favoring by the U.S. Government or any agency thereof. The views and opinions of authors expressed herein do not necessarily state or reflect those of the U.S. Government or any agency thereof. 
(this page intentionally blank) 
INL/EXT-10-20529

November 2010

\title{
Some Specific CASL Requirements for Advanced Multiphase Flow Simulation of Light Water Reactors
}

\author{
R. A. Berry, Ph.D. \\ Idaho National Laboratory
}

\section{Background}

In PWR [Pressurized Water (nuclear) Reactor] safety and optimization there are key issues that rely on in-depth understanding of basic two-phase flow phenomena with heat and mass transfer. Within the context of these multiphase flows, two bubble-dynamic phenomena - boiling (heterogeneous) and flashing or cavitation (homogeneous boiling), with bubble collapse, are technologically very important to nuclear reactor systems. The main difference between boiling and flashing is that bubble growth (and collapse) in boiling is inhibited by limitations on the heat transfer at the interface, whereas bubble growth (and collapse) in flashing is limited primarily by inertial effects in the surrounding liquid. The flashing process tends to be far more explosive (and implosive), and is more violent and damaging (at least in the near term) than the bubble dynamics of boiling. However, other problematic phenomena, such as crud deposition, appear to be intimately connecting with the boiling process. In reality, these two processes share many details, and often occur together.

Flashing occurs in flowing liquid systems when the pressure falls sufficiently low in some region of the flow, reaching a metastable state where the local temperature is higher than the saturated temperature at the reduced pressure of this expanded state. Then the superheated liquid releases its metastable energy (stored as internal energy) very quickly (even explosively), producing either pure vapor (bubble) or liquid-vapor mixture at high velocity. Expansion effects in nuclear reactor systems often occur due to geometrical effects, as for example in flows around solid design features where local flashing and bubble collapse occur or in nozzles where flashing appears at locations where the pressure is relatively low and the liquid superheated. In the case of two-phase blowdown (from the superheated liquid state), bubble collapse is usually not important, but the flashing of superheated liquid strongly influences critical flow rates. In other cases, besides the performance limitations which this flashing (cavitation) may cause in flow systems, subsequent bubble collapse may be responsible for damage to nearby solid surfaces.

Many nuclear reactor applications rely on convective nucleate boiling to efficiently remove high heat fluxes from heated surfaces. Nucleate boiling is a very effective heat transfer mechanism, however it is well known that there exists a critical value of the heat flux at which nucleate boiling transitions to film boiling (Departure from Nucleate Boiling (DNB) and boiling crisis), a very poor heat transfer mechanism. In most practical applications it is imperative to maintain the operating heat flux below such critical value, which is called the 
Critical Heat Flux (CHF). In this case, the presence of a nearby solid surface is necessary for the rapid supply of the latent heat inherent in the phase change. The presence of these surfaces is known to modify the flow patterns and other characteristics of these multiphase flows, and therefore must be interactively coupled with analyses of these phenomena. Again, as mentioned above, DNB is believed to play an integral role in performance degradation as well as the crud deposition problem. Despite several decades of intense study a consensus explanation of the physical mechanism causing CHF is yet to be found, even for the simple situation of pool boiling on a flat plate, let alone flow boiling in a rodbundle geometry, which is the situation of interest in nuclear reactors. Many theories have been formulated, all of which rely on simple CHF models based on an idealized geometry of the vapor/liquid interface. For example, the classic hydrodynamic instability theory of CHF postulates an array of cylindrical vapor jets rising from the heater surface, the macrolayer dryout theory assumes a smooth liquid layer underneath a mushroom-shaped bubble, and the bubble interaction theories typically assume that nucleating bubbles are spherical. However, a very different picture of the physical situation at CHF has been revealed by recent studies [1,2] using sophisticated imaging diagnostics. Briefly, at high heat fluxes there exist numerous dry areas on the surface (with length scales of 2-3 mm in the $\mathrm{x}$ - and $\mathrm{y}$ directions), dispersed within an interconnected network of liquid menisci (with length scales $<50 \mu \mathrm{m}$ in the z-direction). The geometry of the liquid/vapor interface is highly irregular and its nature is dynamic, i.e., the liquid menisci advance into and retreat from the dry areas as a function of time, due to various effects, e.g., liquid inertia (sloshing), capillary forces (surface tension) and recoil forces (evaporation). Recently, good agreement has been obtained by Nikolayev [3] based on the fundamental hypothesis that the boiling crisis is triggered by the vapor recoil when liquid transforms into vapor. Our inability to accurately predict heat transfer and heat transfer regime transitions (flow topologies) in the real nuclear reactor two-phase flow conditions and fuel bundle geometry results in increased safety margins and impedes the development of new fuel designs.

With the advent of increased availability of computational power and of a new generation of methods and codes, the CASL program was recently instituted to foster significant progress in the use of computational fluid dynamics (CFD) and multiphysics methods for nuclear fuel design, thus leading to a drastic reduction in development costs and a justifiable revision of safety margins. As recently observed at the European workshop on "Two-phase Convective Flow Boiling Flow Modelling" [3], our ideas for a broad spectrum of multiphase flow simulation improvements, as will be discussed subsequently, are being echoed by others. In France, CEA, EDF, AREVA and IRSN have launched the NEPTUNE project [4], aimed at providing tools describing two-phase flow and heat transfer that are validated in the parameter range of the industrial applications, plus others covering the entire spectrum of space and time scales. In the U.S., the CASL program has been recently initiated to promote similar collaborative research on this subject at a wide scale.

For example, the two-phase flow phenomena occurring inside a Light Water Reactor (LWR) fuel bundle includes coolant phase changes and multiple flow regimes which directly influence the coolant interaction with the fuel assembly and, ultimately, the reactor performance. The resolution of traditional sub-channel analysis codes is too coarse for analyzing the detailed intra-assembly flow patterns, such as flow around a spacer element 
and it is now generally recognized that their basic modeling approach and computational methods no longer represent state-of-the-art in the field of numerical simulation. New codes need to be developed for the fine-mesh, detailed simulation of LWR fuel assembly two-phase flow phenomena which take advantage of recent progress in Computational Fluid Dynamics (CFD) and the rapidly increasing computational power of massively parallel computers. Similar arguments can be made for the need for high-resolution modeling of localized subcooled boiling and bubble collapse of Pressurized Water Reactors (PWR) to predict performance degradation, especially with regard to the crud deposition problem.

Because modern, high-resolution numerical methods/CFD codes divide the flow space into much finer computational cells it is imperative that we not:

- Utilize traditional multiphase models which are mathematically ill-posed,

- Rely on traditional "flow regime maps" used in subchannel thermal-hydraulics codes to evaluate the interface topology.

Traditional 6-equation, single pressure two-phase mixture models have a state domain in which the square sound speed is negative which produces ill-posedness, non-hyperbolicity, wrong wave dynamics, and inappropriate transient solutions. Such equations have been used in the past because first order numerics were employed on course grids which produced large enough artificial viscosity to render a solution. However, grid convergence with such a scheme is not possible - an untenable situation. Using such equation systems negates the possibility of utilizing modern high-resolution methods (second order or higher), for example, finite volume methods based on Godunov methods with approximate Riemann solvers.

Inter-phase interactions in multiphase fluids depend on both the area and the topology of the interface. Traditional "flow-regime maps" are used in sub-channel thermal-hydraulics codes to evaluate the interface topology from cross-section-averaged flow parameters. Because CFD codes divide the flow space into much finer computational cells, they need not rely on the traditional sub-channel flow regimes. Instead, they must evaluate the local interface surface topology. The advantage realized with this approach is that the ensemble of many computational cells, with relatively simple interface surface topologies, can provide complex global topologies that include all the traditional sub-channel flow regimes. With these simplified flow regime topological maps, which will necessarily depend upon computational cell-size, only a few parameters need be used to determine the interface surface topology in each cell. These parameters will be based on experimental data and direct numerical simulation (DNS) (and DNS-like) of highly resolved phenomena on a small scale, or DNS-like resolved interface simulations on an intermediate scale. 


\section{Direction}

Because of the diversity of physical phenomena occuring in boiling, flashing, and bubble collapse, and of the length and time scales of LWR systems, it is imperative that the models have the following features:

- Both vapor and liquid phases (and noncondensible phases, if present) must be treated as compressible.

- Models must be mathematically and numerically well-posed.

- The models methodology must be multi-scale.

A fundamental derivation of the multiphase governing equation system, that should be used as a basis for advanced multiphase modeling in LWR coolant systems, is given in the Appendix using the ensemble averaging method. For the remainder of this work, focus will be placed specifically on the compressible, well-posed, and multi-scale requirements of advanced simulation methods for these LWR coolant systems. These fundamental aspects are isolated from the general model for further examination here because their correct treatment is key to advanced model/methodology development.

Because of the expense of developing multiple special-purpose codes and the inherent inability to couple information from the multiple, separate length- and time-scales, efforts within CASL should be focused toward development of multi-scale approaches to solve those multiphase flow problems relevant to LWR design and safety analysis. Efforts should be aimed at developing well-designed unified physical/mathematical ${ }^{1}$ and high-resolution numerical models for compressible, all-speed multiphase flows spanning:

(1) Well-posed general mixture level (true multiphase) models for fast transient situations and safety analysis,

(2) DNS (Direct Numerical Simulation)-like models to resolve interface level phenmena like flashing and boiling flows, and critical heat flux determination (necessarily including conjugate heat transfer), and

(3) Multi-scale methods to resolve both (1) and (2) automatically, depending upon specified mesh resolution, and to couple different flow models (single-phase, multiphase with several velocities and pressures, multiphase with single velocity and pressure, etc.) $)^{2}$

\footnotetext{
${ }^{1}$ That is, the effective, nonlinear system of first order partial differential equations.

${ }^{2}$ Specifically, a well-designed 2-pressure, 2-velocity, seven-equation two-phase mixture model (as in 1) can be systematically reduced analytically to produce a 1-pressure, 1-velocity, five-equation model (as for 2) capable of a DNS-like (Direct Numerical Simlation) resolved interface solution. If such a systematic reduction is accomplished numerically on a local spatial level, then a general algorithm (such as 1) can be made to reduce locally where appropriate to the interface resolved model (2), effectively giving an automatic multiscale treatment (as 3).
} 
Such development will extend the necessary foundations and build the capability to simultaneously solve fluid dynamic interface problems as well as multiphase mixtures arising from boiling, flashing or cavitation of superheated liquid, and bubble collapse, etc. in light water reactor systems. It entails development on two main fronts. The first requires the derivation (design) of theoretical models for multiphase and interfacial flows whose mathematical description (equation system) is well-posed and exhibits hyperbolicity, exhibiting correct wave dynamics at all scales. The second requires the design of appropriate numerical schemes to give adequate resolution for all spatial and time scales of interest. These models are not as well known as conventional single-fluid models and pose significant numerical challenges, e.g. the numerical approximation of non-conservative terms. In addition, these numerical issues can pose theoretical questions such as shock wave existence in a multiphase mixture, cell averages of non-conservative variables, etc.

Such two-phase flow phenomena occurring inside light water nuclear reactors includes, especially with departure from nucleate boiling (DNB) and film boiling instability (boiling crisis), coolant phase changes and multiple flow regimes which directly influence the coolant interaction with the fuel elements/assemblies and, ultimately, the reactor performance. Because of the inherent coupling, an understanding of these phemonena, along with subcooled boiling and bubble collapse, is also key to gaining an understanding of crud depostion in these systems [6]. The goal of CASL development needs ultimately to provide models giving highly resolved details where necessary, simultaneously with large scale vessel/component simulation by providing a well-posed, multi-scale model that will:

- Resolve interfaces for larger bubbles (direct numerical simulation, DNS-like) with single velocity, single pressure treatment (interface capturing), and

- Average (or homogenize) the two-phase flow field for small bubbles with two velocities, two pressures.

It is, of course, the aim of CASL to implement the resulting algorithms on modern parallel computing machines for solving large-scale problems for the design and analysis of advanced technology systems such as nuclear energy.

The primary, enabling feature of the INL (Idaho National Laboratory) multi-scale methodology for multiphase flows involves the way in which we deal with multiphase mixtures. Our multiscale approach, which is similar that of the INRIA SMASH group (France) with whom we have collaborated in recent years [5], is essentially to solve the same equations everywhere with the same numerical method:

- In pure fluid,

- In multi-velocity mixtures

- In artificial smearing zones at material interfaces or in mixture cells,

- In phase transition fronts and in shocks,

There are several advantages with this approach:

- Coding simplicity and robustness as a unique algorithm is used; 
- Conservation principles are guaranteed for the mixture. Conventional algorithms are able to preserve mass conservation only when dealing with interfaces;

- Interface conditions are perfectly matched even for the coupling of complex media (capillary fluids, transition fronts) even in the presence of shocks;

- This approach is the only one able to deal with dynamic appearance of interfaces (spontaneous flashing (cavitation) and boiling);

- These methods allow the coupling of multi-velocities, multi-temperature mixtures to macroscopic interfaces where a single velocity must be present. This capability can be illustrated simply by considering the example of a cloud of bubbles rising up in a liquid to the surface, where a free boundary (interface) is present. Two velocities must be considered for the bubbles rising, while a single velocity must be present just after their crossing through the interface. It is also desireable to resolve large bubbles in which a liquid with small bubbles lies outside our large bubble and/or vapor with small droplets lies inside the bubble. This is the only method able to deal with such situations.

Because of the broad spectrum of phenomena occurring in light water nuclear reactor coolant flows (boiling, flashing, and bubble collapse, choking, blowdown, condensation, wave propagation, large density variation convection, etc.) it is imperative that models accurately describe compressible multiphase flow with multiple velocities. The highpressure and/or high-velocity conditions involved in these flows require that compressible effects be considered for all phases (Recalling that effective sound speeds in two-phase mixtures can be as low as 50-80 feet per second, effective Mach numbers well above 0.3 can easily result). Conventional models ${ }^{3}$ of two-phase mixtures having two velocities present are represented with a system of six partial differential equations: two mass, two momentum, and two energy equations. With the assumption of pressure equilibrium, these models have a single pressure common to both phases. These models are not hyperbolic and are ill-posed. This means that initial data and boundary conditions do not fully determine the solution at the next instant in time. Wave propagation may have no physical sense with such systems because the square of the sound speed may become negative.

Baer and Nunziato [9] remedied this problem when they proposed an extended model with seven equations. An additional differential expression (equation) of the pressure

\footnotetext{
${ }^{3}$ The complexity of multiphase, multi-component, and/or multi-material flow dictates that they need to be examined in an averaged sense. Traditionally, one would begin with known (or at least postulated) microscopic flow relations that hold on the "small" scale. These include continuum level conservation of mass, balance of species mass and momentum, conservation of energy, and a statement of the second law of thermodynamics often in the form of an entropy inequality (such as the Clausius-Duhem inequality). The averaged or macroscopic multiphase conservation equations and entropy inequalities are then constructed from the microscopic equations through suitable averaging procedures, as shown in the Appendix. At this stage a stronger form of the second law may also be postulated for the mixture of phases or materials. To render the evolutionary material flow balance system unique, constitutive equations and phase or material interaction relations are introduced from experimental observation, or by postulation, or from microlevel numerical simulation) through strict enforcement of the constraints or restrictions resulting from the averaged entropy inequalities. These averaged equations form the governing equation system for the dynamic evolution of these mixture flows.
} 
equilibrium condition, e.g. [7-12], describing the time evolution of the volume fraction ${ }^{4}$ replaced the pressure equilibrium assumption in the mixture. See the Appendix for details. These terms control the rate at which pressure equilibrium is reached after wave propagation (also as phase velocities equilibrate). Such models have 7- or 8- equations for two-phase flow. (Note: Traditional 6-equation, two-phase models assume both phase have a single pressure, are not hyperbolic, are ill-posed, and give wrong wave dynamics solutions!). With this addition, the model became correctly (well-) posed and unconditionally hyperbolic.

This model had little diffusion (because it was originally presented in the context of a specific problem in detonation physics). The model is difficult to solve numerically, in particular with modern algorithms based on the Riemann problem solution. This issue is especially important for use with DNS-like interface resolving calculations because:

- This model involves two pressures and two velocities,

- At an interface the jump condition corresponds to continuous normal velocities and continuous pressures,

- In order to fulfill this condition it is necessary to relax the two pressures and velocities to unique equilibrium values.

These issues can be resolved by using specific relaxation solvers, with locally infinite relaxation parameters, to solve interface problems and multiphase mixtures with two velocities.

This seven-equation two-phase flow model can be given in slightly different form [17] as

$$
\begin{aligned}
& \frac{\partial \alpha_{1}}{\partial t}+u_{I} \frac{\partial \alpha_{1}}{\partial x}=\mu\left(p_{1}-p_{2}\right)+\frac{\Gamma_{1}}{\rho_{I}} \\
& \frac{\partial\left(\alpha_{1} \rho_{1}\right)}{\partial t}+\frac{\partial\left(\alpha_{1} \rho_{1} u_{1}\right)}{\partial x}=\Gamma_{1} \\
& \frac{\partial\left(\alpha_{1} \rho_{1} u_{1}\right)}{\partial t}+\frac{\partial\left(\alpha_{1} \rho_{1} u_{1}^{2}+\alpha_{1} p_{1}\right)}{\partial x}=p_{I} \frac{\partial \alpha_{1}}{\partial x}-\lambda\left(u_{1}-u_{2}\right)+\Gamma_{1} u_{I} \\
& \frac{\partial\left(\alpha_{1} \rho_{1} E_{1}\right)}{\partial t}+\frac{\partial\left[\alpha_{1}\left(\rho_{1} E_{1}+p_{1}\right) u_{1}\right]}{\partial x}=p_{I} u_{I} \frac{\partial \alpha_{1}}{\partial x}-\mu p_{I}^{\prime}\left(p_{1}-p_{2}\right)-\lambda u_{I}^{\prime}\left(u_{1}-u_{2}\right)+\Gamma_{1} E_{I}
\end{aligned}
$$

for one phase, coupled to the other phase similarly as

\footnotetext{
${ }^{4}$ Specifically, the volume fraction evolution equation is driven, at least in part, by a pressure relaxation effect.
} 


$$
\begin{aligned}
& \frac{\partial \alpha_{2}}{\partial t}+u_{I} \frac{\partial \alpha_{2}}{\partial x}=\mu\left(p_{2}-p_{1}\right)+\frac{\Gamma_{2}}{\rho_{I}} \\
& \frac{\partial\left(\alpha_{2} \rho_{2}\right)}{\partial t}+\frac{\partial\left(\alpha_{2} \rho_{2} u_{2}\right)}{\partial x}=\Gamma_{2} \\
& \frac{\partial\left(\alpha_{2} \rho_{2} u_{2}\right)}{\partial t}+\frac{\partial\left(\alpha_{2} \rho_{2} u_{2}^{2}+\alpha_{2} p_{2}\right)}{\partial x}=p_{I} \frac{\partial \alpha_{2}}{\partial x}-\lambda\left(u_{2}-u_{1}\right)+\Gamma_{2} u_{I} \\
& \frac{\partial\left(\alpha_{2} \rho_{2} E_{2}\right)}{\partial t}+\frac{\partial\left[\alpha_{2}\left(\rho_{2} E_{2}+p_{2}\right) u_{2}\right]}{\partial x}=p_{I} u_{I} \frac{\partial \alpha_{2}}{\partial x}-\mu p_{I}^{\prime}\left(p_{2}-p_{1}\right)-\lambda u_{I}^{\prime}\left(u_{2}-u_{1}\right)+\Gamma_{2} E_{I}
\end{aligned}
$$

where for two phases the second volume fraction equation can be eliminated through the saturation condition $\alpha_{1}+\alpha_{2}=0$. We point out that we have here neglected pressure differences that can be sustained due to relative velocity between the phases, e.g. [13], or due to surface tension effects. We refer to these sustained (or steady-state or static) pressure differences as structural or configuration pressures because they are due to the structure or configuration of the phases. These additional effects can be sustained at steady state - thus for very rapid volume fraction evolution these residual force balances can become an algebraic closure relation. Such pressure differences can be easily accommodated, as appropriate, with modification of the volume fraction evolution equation and the interface pressure expressions in the momentum and energy equations. We neglect these terms here for clarity of presentation and retain only the fastest thermodynamical nonequilibrium terms. For two phases, it holds that $\Gamma_{1}+\Gamma_{2}=0$. In addition to equations of state for each phase ${ }^{5}$ closure relations for this system require the determination of:

- The interface velocity $u_{I}$ and pressure $p_{I}$ representing the velocity and pressure, respectively, that are exerted at the boundary of a cloud of bubbles or droplets,

- The average interface velocity $u_{I}^{\prime}$ and pressure $p_{I}^{\prime}$ that are exerted in the bulk of a two-phase control volume,

- The relaxation parameters $\lambda$ and $\mu$ that control the rate at which velocities and pressures, respectively, relax to mechanical equilibrium.

\footnotetext{
${ }^{5}$ For example stiffened gas equation of state (SGEOS) given for each phase by:

$$
\begin{gathered}
e(p, v)=\frac{p+\gamma p_{\infty}}{(\gamma-1)} v+q \\
v(p, T)=\frac{(\gamma-1) C_{v} T}{p+p_{\infty}} \\
h(T)=\gamma C_{v} T+q \\
g(p, T)=\left(\gamma C_{v}-q^{\prime}\right) T-C_{v} T \log \frac{T^{\gamma}}{\left(p+p_{\infty}\right)^{(\gamma-1)}}+q
\end{gathered}
$$
}


Originally, these relations were unknown, either estimated in limiting cases only, or determined by experimental means. Also, with conventional numerical approaches, the resulting partial differential equation (PDE) system (7), non-conservative and needing nontrivial closure relations, is integrated numerically over space and time. This presents an additional problem related to the numerical approximation of the non-conservative terms. The numerical approximation of these models is a difficult task, not only because they contain non-conservative terms, but also because they exhibit many types of waves. Up to seven waves are present for correctly formulated models (a set of two acoustic waves and a contact discontinuity are present for each fluid plus a volume fraction wave representing a possible phase change front). These wave patterns increase the complexity of computation of the fluxes. As mentioned, the presence of non-conservative terms poses difficulties, especially with the occurrence of discontinuities such as interfaces and shocks [14-16]. However, in order to determine the closure relations indicated above and to cirumvent these difficulties of the seven-equation PDE models [7-12], a new homogenization method was constructed in [17].

This new averaging method, termed the Discrete Equation Method (DEM) ${ }^{6}$, considers the mixture at the discrete level, with a stencil composed of three computational cells (in 1-D). In each cell, at each cell boundary and at each internal boundary separating the phases, the Riemann problem (RP) of the pure fluid equations is solved. The RP solution provides all local interfacial information. These RP solutions are then averaged in the computational cell as done originally with the first version of the Godunov method, derived originally for the Euler equations. In this context, the additional difficulties that occur, due to the presence of internal material interfaces, material discontinuities at cell boundaries, and variable sub-volumes, because of the phase presence in the cells, are addressed. The philosophy, however, remains the same as with the Godunov method - the average RP solutions are dealt with directly, not with discretized partial differential equations.

\footnotetext{
${ }^{6}$ The discrete equation method (DEM) is not only a homogenization method but is in fact a numerical method that applies the fundamental ideas of the Godunov method to a two-phase control volume [18-20]. The DEM proceeds in the opposite way to what is conventionally done (as described above). The solutions for the pure fluid equations are computed at the scale of each inclusion, and then these solutions are averaged over the fluids' volumes inside the computational cell. More specifically, the pure phase conservation laws are considered at the microscopic level. The method involves solving in every cell for each two-phase control volume, at its boundaries and at its internal interfaces, an initial value problem between the various fluids and their states. The solution of these interface problems is obtained using Riemann (or approximate Riemann) solvers for the Euler equations. In fact, each pure fluid is governed by the Euler equations. All Riemann problem solutions are then averaged over the phases control volumes, similarly as done with the Euler equations and the Godunov scheme, to provide the corresponding numerical scheme for the averaged multiphase flow equations. Indeed the result is a set of discrete equations that describes the two-phase mixture involving interaction terms. These discrete equations correspond to a numerical scheme - one which differs only slightly from traditional single phase, finite volume Euler solution methods. This model is hyperbolic and well-posed. Moreover, not only does the method provide the numerical scheme, but it implicitly contains the correct averages of interfacial pressure and velocity as well as relaxation terms [19]. In fact, if acoustic Riemann solvers [Godunov] (weak wave assumption) are used and the limit is taken as the cell size and time step go to zero, the 7-equation models are obtained with closure relations! (This can be very useful for some theoretical considerations.)
} 
The resulting system of this averaging procedure is a rather complicated discrete system in algebraic form, corresponding to the result of the Discrete Equations Method (DEM). Though more general than the 7-equation model detailed above, the DEM model surprisingly converges to the 7-equation model as $\Delta x, \Delta t \rightarrow 0$ under the weak wave assumption, but with the added bonus that the closure relations for the various interface variables are obtained, providing information which is easier to interpret than discrete formulas [26, 27]. This approach has recently been applied to fully nonequilibrium twophase, steam-water nozzle flows in [20], wherein it was demonstrated that the DEM approach could be integrated to stable steady state, an important capability when considering LWR systems.

The Direct Numerical Simulation (DNS) or DNS-like simulation of interfacial flows is of fundamental importance for the understanding and prediction of heat transfer between fuel rods and coolant; DNS can be a valuable tool to predict critical heat flux, especially at high-pressure conditions ${ }^{7}$. In order to solve problems wherein it is desired to resolve interfaces separating pure fluids or pure materials (DNS-like simulations), the nonequilibrium flow model with two pressures and two velocities is solved with relaxation of these variables locally, in the vicinity of an interface, to equilibrium values (by setting $\lambda$ and $\mu$ to large values). More specifically,

$$
\mu, \lambda=\left\{\begin{array}{lr}
+\infty & \text { if } \varepsilon \leq \alpha_{1} \leq 1-\varepsilon \\
0 & \text { otherwise }
\end{array}\right.
$$

where $\varepsilon$ is a small parameter, e.g. $10^{-8}$.

This method resolves (captures, see below) interfaces with excellent fulfillment of interface conditions in mixture cells that appear because of numerical diffusion at material interfaces. Develop is in progress of an efficient all-speed or implicit time scheme for the DEM method.

The multiscale aspects of light water nuclear reactor flows dictate the need to be able to resolve interfaces of a specified size while homogenizing interface structures with sizes below this specification. Advanced CASL methods will need to be able to accurately resolve interface problems in extreme flow conditions (high pressure ratios and high density ratios $\approx 10^{3}$ ), as well as compute the dynamic appearance of interfaces (interfaces occuring from spontaneous generation of a phase not initially present), in a computationally efficient manner. Moreover, the formulation and algorithm must be able to not only

\footnotetext{
${ }^{7}$ Presently there is no credible simulation model or code able to deal with DNS of boiling flows. The literature provides a few references dealing with this. For example in S. Shin and D. Juric, "Modelling threedimensional flow using level contour reconstruction method for front tracking without connectivity," J. Comp. Phys. 169 (2002) 503-555, two sets of incompressible Navier-Stokes equations are coupled with a front tracking algorithm where jump conditions of mass, momentum, and energy are set. However, with each fluid being considered incompressible, the volume rate of change of bubbles due to phase change is only approximate. With such an approach, volume changes due to buoyancy effects cannot be correctly accounted for. Lastly, contact angle modeling was not addressed.
} 


\section{capture interfaces separating pure media but also capture interfaces separating} mixtures of fluids in which wave dynamics is also important. The DEM method can resolve two-phase interfaces in a DNS-like manner if sufficient grid resolution is provided, however it can be more sophisticated than is necessary when two-velocity mixtures are not present on either side of the resolved interface. Because, in theory, an interface can be resolved in a diffuse manner (with arbitrarily determined thickness) with complete thermodynamics and with only a single-velocity, single-pressure, 2-temperature two-phase model, attention will be focused next on consistently reduced models for this purpose.

This other option (within the unified hierarchy) consists in determining the asymptotic model resulting from stiff mechanical relaxation, in various forms $[28,29,30]$. In the context of two fluids, this results in a reduced set of five partial differential equations: two phasic mass, one mixture momentum, one mixture energy, and one volume fraction equation. This five-equation system includes the minimal physics necessary to provide complete thermodynamics of each phase along with the interphase transition thermodynamics to satisfy the outlined requirements. This system is less general than the previous non-equilibrium system, but it is of particular interest for resolving interface problems, where a single velocity is present. More precisely, it can be more appropriate and simple, when considering extra physics extensions such as phase transition, capillary effects, etc.

Unlike more common approaches, there is no need to use an Interface Tracking Method (ITM), nor level set, nor interface reconstruction, etc. The same equations are solved

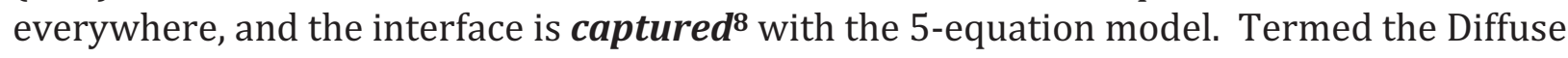
Interface Method (DIM), this model provides correct thermodynamic variables in artificial mixture zones. It permits automatic merging, break-up, coalescence and extreme deformation of interfaces. Though seemingly artificial, this model can handle very large density ratios, and materials governed by very different equations of state, in multidimensions [21]. It is also able to describe multiphase mixtures where stiff mechanical relaxation effects are present.

The reduced, 5-equation model is obtained from the seven-equation model above by forcing mechanical equilibrium, i.e. pressure and velocity relaxation ${ }^{9}$ are obtained by taking the asymptotic limit, respectively, as $\mu \rightarrow \infty$ and $\lambda \rightarrow \infty$ [22]. With inclusion of heat and mass transfer between the phases, this yields:

\footnotetext{
${ }^{8}$ In gas dynamics, the debate about tracking methods (at that time "shock-fitting") versus capturing came about 40 years ago. The development of methods with limited artificial viscosity (Godunov type schemes) and the increase in computational resources ended the debate in the 1980's with the definite success of the capturing methods.

${ }^{9}$ An illustrative example of the non-intuitive solution behavior that can accompany even simple relaxation problems is given in R.J. LeVeque, Finite Volume Methods for Hyperbolic Problems, Cambridge University Press, UK, 2002.
} 


$$
\begin{gathered}
\frac{\partial \alpha_{1}}{\partial t}+\nabla \alpha_{1} \cdot u=\frac{\left(\rho_{2} c_{2}^{2}-\rho_{1} c_{1}^{2}\right)}{\frac{\rho_{1} c_{1}^{2}}{\alpha_{1}}+\frac{\rho_{2} c_{2}^{2}}{\alpha_{2}} \nabla \cdot u+\frac{\left(\frac{\Gamma_{1}}{\alpha_{1}}+\frac{\Gamma_{2}}{\alpha_{2}}\right)}{\frac{\rho_{1} c_{1}^{2}}{\alpha_{1}}+\frac{\rho_{2} c_{2}^{2}}{\alpha_{2}}} Q_{1}+\frac{\frac{c_{1}^{2}}{\alpha_{1}}+\frac{c_{2}^{2}}{\alpha_{2}}}{\frac{\rho_{1} c_{1}^{2}}{\alpha_{1}}+\frac{\rho_{2} c_{2}^{2}}{\alpha_{2}}} \rho \dot{Y}_{1}} \\
\frac{\partial \alpha_{2} \rho_{2}}{\partial t}+\nabla \cdot \alpha_{2} \rho_{1} u=\rho \dot{Y}_{1} \\
\frac{\partial \rho u}{\partial t}+\nabla \cdot(\rho u \otimes u+p I)=0 \\
\frac{\partial \rho E}{\partial t}+\nabla \cdot u(\rho E+p)=0
\end{gathered}
$$

where

$$
\begin{gathered}
e=Y_{1} e_{1}\left(\rho_{1}, p\right)+Y_{2} e_{2}\left(\rho_{2}, p\right) \\
Y_{k}=\frac{(\alpha \rho)_{k}}{\rho} \\
\rho=(\alpha \rho)_{1}+(\alpha \rho)_{2}
\end{gathered}
$$

are the mixture internal energy, the phasic mass fractions, and the mixture internal energy, respectively. Each fluid is governed by its own convex equation of state (EOS)

$$
e_{k}=e_{k}\left(\rho_{k}, p\right)
$$

which allows the determination of the phasic sound speeds

$$
c_{k}=c_{k}\left(\rho_{k}, p\right)
$$

The mixture or equilibrium pressure $p$ is determined for the particular case of fluids governed by the stiffened gas EOS,

$$
p_{k}=\left(\gamma_{k}-1\right) \rho_{k}\left(e_{k}-q_{k}\right)-\gamma_{k} p_{\infty k}
$$

for which the resulting mixture EOS reads

$$
p\left(\rho, e, \alpha_{1}, \alpha_{2}, Y_{1}, Y_{2}\right)=\frac{\rho\left(e-Y_{1} q_{1}-Y_{2} q_{2}\right)-\left(\frac{\alpha_{1} \gamma_{1} p_{\infty 1}}{\gamma_{1}-1}+\frac{\alpha_{2} \gamma_{2} p_{\infty 2}}{\gamma_{2}-1}\right)}{\frac{\alpha_{1}}{\gamma_{1}-1}+\frac{\alpha_{2}}{\gamma_{2}-1}} .
$$


The development above has employed Gruneisen coefficients $\Gamma_{k}=\frac{1}{\rho_{k}}\left(\frac{\partial p_{k}}{\partial e_{k}}\right)_{\rho_{k}}$ for each phase, interface density $\rho_{I}=\frac{\frac{\rho_{1} c_{1}^{2}}{\alpha_{1}}+\frac{\rho_{2} c_{2}^{2}}{\alpha_{2}}}{\frac{c_{1}^{2}}{\alpha_{1}}+\frac{c_{2}^{2}}{\alpha_{2}}}$, and interface temperature $T_{I}=\frac{\frac{\Gamma_{1} T_{1}}{\alpha_{1}}+\frac{\Gamma_{2} T_{2}}{\alpha_{2}}}{\frac{\Gamma_{1}}{\alpha_{1}}+\frac{\Gamma_{2}}{\alpha_{2}}}$. The interphase mass and heat transfers are given, respectively, by

$$
\begin{aligned}
& \dot{Y}_{1}=v\left(\bar{g}_{2}-\bar{g}_{1}\right) \\
& Q_{1}=H\left(T_{2}-T_{1}\right)
\end{aligned}
$$

where $\bar{g}_{k}=h_{k}-\bar{T}_{k}$ are the extended Gibbs free energies formed with temperature $\bar{T}=\frac{T_{1} T_{2}}{T_{I}}$. Obviously, $v$ and $H$ can be treated as thermodynamic relaxation parameters.

To demonstrate the multidimensional capability as well as the dynamic creation of interfaces, a two-dimensional test, involving a Richtmyer-Meshkov instabilitly (RMI), is presented from [21]. The liquid is not pure and new interfaces will appear during development of the instability due to cavitation effects. The shape of the resulting interface and the entire flow field show a non-conventional behavior, never computed before, as the model and method must deal with liquid-gas interfaces and the dynamic appearance of gas pockets in severe conditions. The physical domain is $3 \mathrm{~m}$ long and $1 \mathrm{~m}$ high, with the left part of the computational domain filled with nearly pure water and the right part with nearly pure gas. They are initially separated by a curved interface, a portion of a circle with $0.6-\mathrm{m}$ radius centered at $\mathrm{x}=1.2 \mathrm{~m}, \mathrm{y}=0.5 \mathrm{~m}$. The mesh contains 900 cells in the $\mathrm{x}$-direction and 400 cells in the y-direction. Both water and gas have an initial pressure of 1 atmoshere and an initial velocity of $-200 \mathrm{~m} / \mathrm{s}$ (to the left). The initial density of water and gas is 1000 $\mathrm{kg} / \mathrm{m}^{3}$ and $100 \mathrm{~kg} / \mathrm{m}^{3}$, respectively. Top, bottom, and left boundaries are treated as solid walls; right boundary is open. Both phases are treated with the stiffened gas equation of state; therefore, both phases are assumed compressible. The water in the left part contains a very small volume fraction of gas, $10^{-6}$, while the gas in the right part contains a very small volume fraction of water, $10^{-6}$. The initial configuration is shown in the upper graphic of Figure 1, and the mixture density contours at times $0.0,1.9,3.9,5.8$, and $7.8 \mathrm{~ms}$ in the other graphics of the figure. When flow impacts the left wall, a right-traveling wave propagates in the domain through the water/gas discontinuity. A conventional RMI appears first. Then expansion waves are produced as the jet elongates. It results in expanded zones near the solid boundary where gas inhomogeneities grow, producing the dynamic appearance of gas pockets (white regions at the left boundary) and interfaces. Because the pressure is very low in these zones, the jet's dynamics are modifed from the conventional RMI in pure fluids. The various gas pockets near the solid boundary and in the jet core are clearly visible in Figure 1. Relaxation terms present in the volume fraction 
Nearly pure liquid $\left(\alpha_{\text {gas }}=10^{-6}\right)$
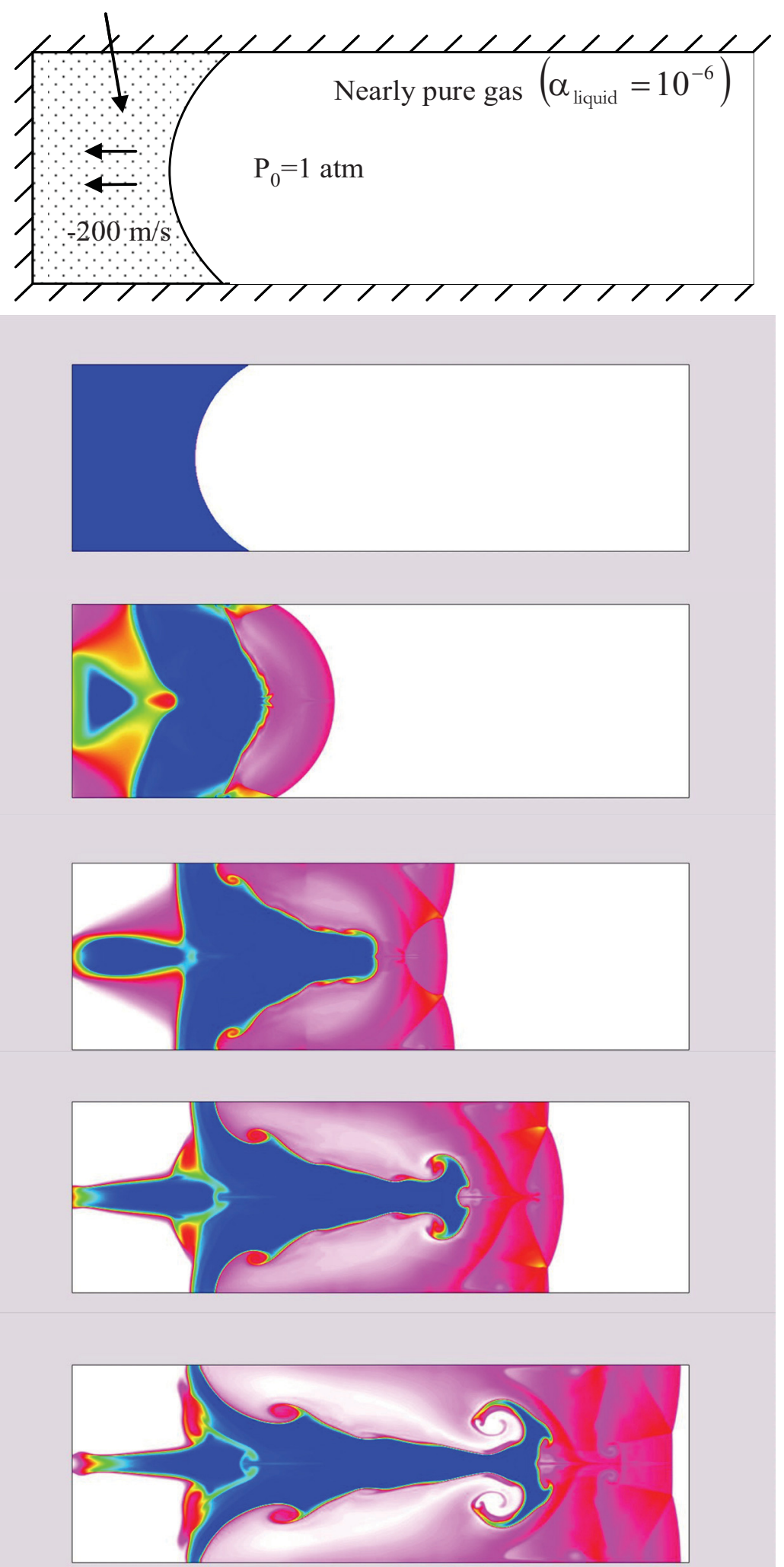

Figure 1. Configuration and mixture density contours at 0.0, 1.9, 3.9, 5.8, and $7.8 \mathrm{~ms}$. Blue is high density, white is low, and others colors are intermediate. 
and energy equations are responsible for the dynamic appearance of these gas pockets. This would be very difficult to accomplish with current sharp interface tracking methods.

This hyperbolic, well-posed system with five equations connects two limit models: A mechanical equilibrium model responsible for acoustic propagation and formation of metastable states, and a thermodynamic equilibrium model to enforce interface conditions. The model involves five waves: $u, u \pm c_{w}$, and $u \pm c_{e q}$ that occur because of relaxation terms $v$ and $H$. Obviously, if we set these relaxation functions to zero (no relaxation, i.e. there is no interphase mass and heat transfer) this strictly hyperbolic system reproduces propagation of acoustic disturbances with characteristic wave speeds, $u$ (3fold), $u+c_{w}$, and $u-c_{w}$ where $c_{w}$ is the Wood speed of sound (mixture)

$$
\frac{1}{\rho c_{w}^{2}}=\frac{\alpha_{1}}{\rho_{1} c_{1}^{2}}+\frac{\alpha_{2}}{\rho_{2} c_{2}^{2}}
$$

This sound speed has a non-monotonic behavior versus volume fraction. More will be said of the thermodynamic equilibrium sound speed, $c_{e q}$, subsequently (footnote 10).

The general determination of the temperature relaxation parameter $H$ for a two-phase mixture with arbitrary interfacial area is a difficult issue, as also is that of the phase transition kinetics parameter $v$, which depends not only on interfacial area but also on local chemical relaxation (see five-equation model). To circumvent these difficulties it is advantageous to choose these as infinite relaxation parameters, i.e. $H, v \rightarrow+\infty$, but only locally so as to retain metastable states. For example, in order to retain metastable states, the relaxation parameters $v$ and $H$ can be set to zero for locations far from the interfaces. At the interfaces they can be taken infinitely large, in order to fulfill equilibrium interface conditions with mass transfer

$$
v, H=\left\{\begin{array}{lr}
+\infty & \text { if } \varepsilon \leq \alpha_{1} \leq 1-\varepsilon \\
0 & \text { otherwise }
\end{array}\right.
$$

where, again, $\varepsilon$ is a small parameter, e.g. $10^{-8}$. Interestingly, if this thermodynamic equilibrium limit was taken globally, i.e. temperature and Gibb's energy relaxation are obtained by taking the asymptotic limit, respectively, as $H \rightarrow \infty$ and $v \rightarrow \infty$, the threeequation homogeneous equilibrium model would result ${ }^{10}$.

\footnotetext{
${ }^{10}$ This relaxation method corresponds to the following (possibly locally, at interface only) limit system, corresponding to the mixture Euler equations

$$
\begin{gathered}
\frac{\partial \rho}{\partial t}+\nabla \cdot(\rho \vec{u})=0 \\
\frac{\partial \rho \vec{u}}{\partial t}+\nabla \cdot(\rho \vec{u} \otimes \vec{u}+p I)=0
\end{gathered}
$$
}


The situation is more difficult with bubbly flows because the interface is not a simple contact surface (as in gas dynamics). Heat and mass transfer are present, rendering the interface permeable, i.e. the fluids on each side of the resolved interface are not pure, they are two-phase with a smaller, unresolved structural scale. Moreover, these transfers are coupled to capillary and dissipative effects. Capturing this type of interface is not only a numerical issue, but also a modeling issue. Progress has recently been achieved in this direction.

A diffuse interface model of compressible capillary fluid was derived and solved in [31]. Key points of this model compared to Cahn and Hilliard [32] type models is that no capillary length is introduced (no special resolution of the interface is needed) and

$$
\frac{\partial \rho E}{\partial t}+\nabla \cdot[(\rho E+p) \vec{u}]=0
$$

where the mixture density is $\rho=\alpha_{1} \rho_{1}+\alpha_{2} \rho_{2}$ and the mixture internal energy is $\rho e=\alpha_{1} \rho_{1} e_{1}+\alpha_{2} \rho_{2} e_{2}$. The total mixture energy is still defined by $E=e+\frac{\vec{u} \cdot \vec{u}}{2}$.

This system is closed by the three thermodynamic equilibrium conditions

$$
\begin{gathered}
p_{1}=p_{2}=p \\
T_{1}=T_{2}=T \\
g_{1}=g_{2}
\end{gathered}
$$

With the pressure and temperature equilibrium conditions above and with the help of the SGEOS, each phase variable can be expressed as a function of pressure and temperature. The definitions of mixture density and internal energy become

$$
\begin{gathered}
\rho=\alpha_{2}\left[\rho_{2}(p, T)-\rho_{1}(p, T)\right]+\rho_{1}(p, T) \\
e=\frac{1}{\rho}\left\{\alpha_{2}\left[\rho_{2}(p, T) e_{2}(p, T)-\rho_{1}(p, T) e_{1}(p, T)\right]+\rho_{1}(p, T) e_{1}(p, T)\right\}
\end{gathered}
$$

The equality of phasic Gibb's energies above reduces the mixture EOS and this system to

$$
\begin{gathered}
\alpha_{2}(T)=\frac{\rho-\rho_{1}(T)}{\rho_{2}(T)-\rho_{1}(T)} \\
e(T)=\frac{1}{\rho}\left\{\alpha_{2}(T)\left[\rho_{2}(T) e_{2}(T)-\rho_{1}(T) e_{1}(T)\right]+\rho_{1}(T) e_{1}(T)\right\}
\end{gathered}
$$

This last equation can be numerically solved for $T$ (substitute the first equation into the second for $\alpha_{2}$ ), given $\rho$ and $e$ from the PDE system solution. It allows the determination of $\alpha_{2}$ with the first equation and of $p$ with the mixture EOS. Thus, this system is closed. By using the sound speed definition $c^{2}=(\partial p / \partial \rho)_{s}$ the following expression is obtained for the thermodynamic equilibrium mixture speed of sound:

$$
\frac{1}{\rho c_{e q}^{2}}=\frac{\alpha_{1}}{\rho_{1} c_{1}^{2}}+\frac{\alpha_{2}}{\rho_{2} c_{2}^{2}}+\left[\frac{\alpha_{1} \rho_{1}}{C_{p, v}}\left(\frac{d s_{1}}{d p}\right)^{2}+\frac{\alpha_{2} \rho_{2}}{C_{p, l}}\left(\frac{d s_{2}}{d p}\right)^{2}\right] .
$$

Note the Wood formula is recovered with the first two terms of this expression. This limit model is again hyperbolic with the characteristic wave speeds $u+c_{e q}, u-c_{e q}$, and $u$. Obviously, $c_{e q}<c_{w}$. An interesting physical observation can also be made: The evaporation front speed in metastable liquids corresponds to those of acoustic waves of the relaxed (thermodynamic equilibrium) system. 
arbitrarily large density ratios at interfaces can be considered ${ }^{11}$. The heat and mass transfer at interfaces resulting in phase transitions were solved in the context of diffuse interfaces in [22]. The main issue was related to the thermodynamic closure which is usually done with van der Waals type equation of state in Cahn-Hilliard-Korteweg theory. With this theory, phase transition corresponds to a thermodynamic transformation, which results in two inconsistencies:

- The phase transition time is absent in this description, and

- When capillary effects are absent, the square sound speed may become negative, resulting in ill-posedness.

With the 5-equation, diffuse interface capturing approach, phase transition corresponds to a kinetic transformation. It uses non-equilibrium and equilibrium thermodynamic considerations, but the key point is the kinetic representation that solves the two preceding issues. Incorporation of interfacial surface tension and heat and mass transfer effects gives a simple, efficient and robust method that can be used to resolve fundamental boiling issues in a DNS-like manner similar to the cavitation example above. Furthermore, the various components employed in this method are general enough to merit coupling with additional complex physics and chemistry, e.g. to address the unwanted deposit of constituents dissolved in the coolant as a result of localized boiling -- the so-called crud deposition problem. An excellent example using the diffuse interface method to resolve interfaces with phase change due to intense flashing, and with inclusion of a third, noncondensing phase was shown recently in [33].

Figure 2 illustrates the hierarchy of the unified, consistent relationships between the generalized DEM, reduced models, and DIM methodologies used for multiphase flows with consistent mixture homogenization and interface capturing treatment. The most general DEM method begins in the top left corner. Immediately below that is indicated that if the limit is taken as the grid parameters go to zero, and a weak wave or acoustic Riemann solver is used to relate differential quantities, the fully nonequilibrium seven-equation PDE, with non-conservative terms results. Progressing down the left column with successively more relaxation parameters being globally taken as numerically large, produces consistenly reduced models for homogenized mixtures - the five-equation PDE system in mechanical equilibrium, then the three-equation homogeneous equilibrium model in both mechanical and thermal equilibrium. If the various relaxation parameters are relaxed only locally, specifically in the neighborhood of the interface, then DIM models result for resolving an interface via capturing, as shown in the corresponding right column.

CASL research should be ultimately aimed at developing unified and well-designed physical/mathematical models along with high-resolution numerical solution methods for general multi-scale, multiphase flow. To test this approach, thereby reducing the uncertainties of implementation, in recent INL internal reasearch efforts, analytically

\footnotetext{
${ }^{11}$ See Jamet, D., Lebiague, O., Coutris, N., and Delhaye, J.M.,"'The second gradient method for the direct numerical simulation of liquid-vapor with phase change," J. Comp. Phys. 169 (2001) 624-651, where very limited density ratios are considered.
} 


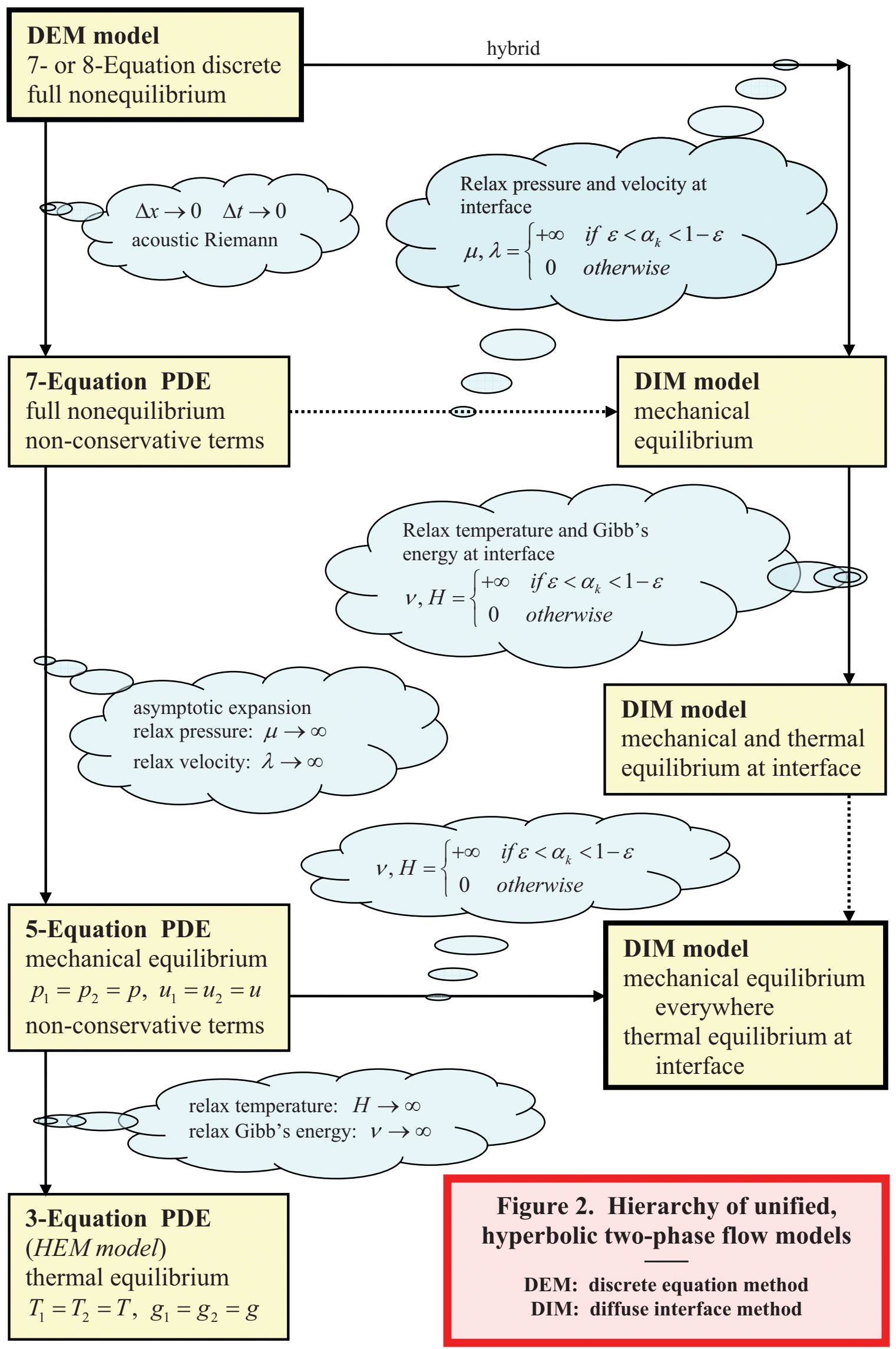


reduced models (using asymptotic expansions) of the general mixture equations were built and used to produce DNS-like, interface-resolving solutions for multiple compressible fluid phases. Because of the inherent weaknesses of sharp interface methods (SIM), e.g. level-set methods, for DNS-like simulations, most notably their inability to dynamically create interfaces and to solve interfaces separating pure media and mixtures, we focused on a DIM which does not exhibit these weaknesses. As discussed above, the DIM considers interfaces as numerically diffused zones corresponding to artificial mixtures created by numerical diffusion. The determination of thermodynamic flow variables in these zones is achieved on the basis of multiphase flow theory. The challenge, however, was to derive physically, mathematically, and numerically consistent thermodynamic laws for the artificial mixture of the diffused interface which would fulfill correct interface conditions. INL research works in this area are documented in [21]. A huge advantage of this approach is that the same algorithm is implemented globally in both pure fluids and in mixture zones. For this research, a single velocity, non-conservative hyperbolic model was developed, with two energy equations involving relaxation terms, which fulfills the equation of state and energy conservation on both sides of interfaces and guarantees correct transmission of pressure waves across them. This formulation considerably simplifies numerical resolution within the context of diffuse interfaces. Codes have been constructed to successfully demonstrate this methodology [21-24].

Multiphase formulations have been developed to give the ability to solve problems involving both heterogeneous mixtures of materials and interfacial flows involving

compressibility and phase transition. In particular, for the DNS-like simulation of interfacial flows, of prime importance for the nuclear industry for reactor safety and optimization, the aim is to compute the critical heat flux conditions that involve a competition between bubble growth, surface tension, contact angle effects, heat and mass transfers at interfaces. Contrary to the approach employed with other ITM methodologies, the approach espoused here embraces a general model that accounts for complete thermodynamics in both phases.

All of the methods discussed or demonstrated above have been successfully demonstrated at the INL by utilizing explicit time integration. Under CASL, two major improvements must occur:

(1) The methods must be employed with some kind of a preconditioner to allow accurate low Mach number solutions (especially for liquid phases) and, indeed, allspeed solutions ${ }^{12}$. An initial investigation of the extension of the DIM to an all-speed

\footnotetext{
${ }^{12}$ The models of the type described in this report, with volume fraction evolution equation containing terms proportional to simple phasic pressure difference normalized by a coefficient time scale, will reduce to the corresponding single-pressure model in the incompressible limit (for both phases). These models are essentially compressible in nature and equilibrate with an acoustic time scale. As written, they cannot describe a residual interphase pressure difference that may persist in the much slower non-acoustic or even incompressible limit. If such pressures are believed significant, the volume fraction evolution equations need only be modified to include time scales for equilibration determined by inertial rather than (or in addition to) acoustic effects. Irrespective of the time scale, the presence of terms proportional to pressure differences is
} 
relaxation scheme for interface flows with surface tension has been recently reported in [35].

(2) To relieve time step restrictions due to stiffness and to achieve tighter coupling of equations, all methods need to evolve with implicit time integration.

With the construction of these methods utilizing implicit time integration, numerical integration of the stiff relaxation terms can be performed (thereby bypassing the asyptotically reduced models) to produce a truly multi-scale, unified method which can handle two-phase mixtures at the large, or under resolved scale, and DNS-like two-phase modeling on the small, or highly resolved, scale. Simple, efficient and robust algorithms will be developed to solve the well-posed models. The various ingredients employed in these methods will be general enough to accommodate extensions to enlarged problems involving complex multiphysics. Using the fractional step methods typically applied, and miss-applied, to calculate these types of initial value problems, that consist of different kinds of physics with multiple time-scales, some of which should be treated implicitly, requires some precautionary measures to avoid splitting and conditioning errors. Appropriate fully implicit methods must be pursued which will allow integration over the fast time scales for slow speed flows using a Jacobian-Free Newton-Krylov method with physics based preconditioning to allow tightly coupled solutions of the multiphysics phenomena, e.g. as in [25], inherent in nuclear reactor core applications.

\section{References}

1. Theofanus T.G. et al., "The Boiling Crisis Phenomena. Part II: Dryout Dynamics and Burnout," Experimental Thermal and Fluid Science, 26, 793-810 (2002).

2. Nishio S. and H. Tanaka, "Visualization of Boiling Structures in High-Heat Flux PoolBoiling," Int. J. Heat Mass Transfer, 47, 4559-4568 (2004).

3. "Two-phase Convective Flow Boiling Flow Modelling", organized by the Societe Hydrotechnique de France (SHF) and the International Association of Hydraulic Engineering and Research (IAHR) under sponsorship of Societe Francaise des Thermiciens (SFT), CEA, AREVA, EDF, and AFM. Workshop was held in Grenoble, France, Sept. 8-9, 2008.

4. A. Guelfi, D. Bestion, M. Boucker, P. Boudier, P. Fillion, M. Grandotto, J.M. Herard, E. Hervieu, and P. Peturaud, "NEPTUNE: a new software platform for advanced nuclear thermal hydraulics," Nuclear Sci. and Eng. 156 (2007) 281-324.

essential to describe the tendency for the pressures to equilibrate. Models that lack such terms therefore neglect an essential aspect of the physics [34]. If such effects equilibrate on a time scale very rapid in comprarison to the convective time scales, then appropriate equilibrium relations may substitute for the volume fraction evolution equation. 
5. R.A. Berry, R. Saurel, F. Petitpas, E. Daniel, O. LeMetayer, S. Gavrilyuk, N. Dovetta, and R.C. Martineau, "Progress in the development of compressible, multiphase flow modeling capability for nuclear reactor flow applications," Idaho National Laboratory Report (2008) No. INL/EXT-08-15002.

6. R.A. Berry, and S. Kadioglu, "High-Resolution, Two-phase Flow Modeling Challenges for Light Water Nuclear Reactors," 2008 SIAM Annual Meeting, San Diego, CA, July 2008.

7. V.H. Ransom and M.P. Scofield, "Two-pressure hydrodynamic model for two-phase separated flow," Idaho National Engineering Laboratory Report (1976) No. SRD-5076.

8. H. Nguyen, "One-dimensional models for transient two-phase separated flow," $3^{\text {rd }}$ CSNI Specialist Meeting on Transient Two-Phase Flow," California Institute of Technology, Pasadena, California, March 23-25, 1981.

9. M.R. Baer and J.W. Nunziato, "A two-phase mixture theory for the deflagration-todetonation transition (ddt) in reactive granular materials," Int. J. Multiphase Flow 12 (1986) 861-889.

10. R. Saurel, A. Forestier, D. Veyret, and J.-C. Loraud, “A finite volume scheme for twophase compressible flows,” Int. J. Num. Meth. Fluids 18 (1994) 803-819.

11. M.-H. Lallemand and R. Saurel, "Pressure relaxation procedures for multiphase compressible flows," INRIA Research Report (2000) No. 4038.

12. C.H. Chang and J.D. Ramshaw, "Dynamical evolution of volume fractions in multipressure multiphase flow models," Phys. Rev. E 77 (2008) 066305-1-7.

13. D.A. Drew and S.L. Passman, Theory of Multicomponent Fluids, Springer-Verlag, New York, 1999.

14. G. Dalmaso, P.G. LeFloch, and F. Murat, "Definition and weak stability of nonconservative products," J. Math. Pures Appl. 74 (1995) 483-548.

15. C. Pares, "Numerical methods for nonconservative hyperbolic systems: a theoretical framework," SIAM J. Numer. Anal. 44 (2006) 300-321.

16. R. Abgrall and S. Karni, "A comment on the computation of non-conservative products," J. Comput. Phys. 229 (2010) 2759-2763.

17. R. Abgrall and R. Saurel, "Discrete equations for physical and numerical compressible multiphase mixtures," J. Comput. Phys. 186 (2003) 361-396. 
18. 0. LeMetayer, J. Massoni, and R. Saurel, "Modelling evaporation fronts with reactive Riemann solvers," J. Comput. Phys. 205 (2005) 567-610.

19. R. Saurel, J. Massoni, and F. Renaud, "A numerical method for one-dimensional compressible multiphase flows on moving meshes," Int. J. Num. Meth. Fluids 54 (2007) 1425-1450.

20. R.A. Berry, R. Saurel, O. LeMetayer, "The discrete equation method (DEM) for fully compressible, two-phase flows in ducts of spatially varying cross-section," Nuclear Engineering and Design 240 (2010) 3797-3818.

21. R. Saurel, F. Petitpas, and R.A. Berry, "Simple and efficient relaxation methods for interfaces separating compressible fluids, cavitating flows and shocks in multiphase mixtures," J. Comput. Phys. 228 (2009) 1678-1712.

22. R. Saurel, F. Petitpas, and R. Abgrall, "Modeling phase transition in metastable liquids: application to cavitating and flashing flows," J. Fluid Mech. 607 (2008) 313350.

23. F. Petitpas, J. Massoni, R. Saurel, E. Lapebie, and L Munier, "Diffuse interface model for high speed cavitating underwater systems," Int. J. Multiphase Flow 35 (2009) 747-759.

24. R.A. Berry, R. Saurel, and F. Petitpas, "Unified two-phase CFD modeling of boiling, cavitation, and bubble collapse," Fluids Engineering (Spring 2009) 10-11.

25. S. Kadioglu and D. Knoll, "A fully second order implicit/explicit time integration technique for hydrodynamic plus nonlinear conduction problems," J. Comput. Phys. 229 (2010) 3237-3249.

26. Saurel, R., Gavrilyuk, S., Francois. Renaud, "A multiphase model with internal degree of freedom : Application to shock bubble interaction," Journal of Fluid Mechanics 495 (2003) 283-321.

27. Chinnayya, A., Daniel, and E., Saurel, R., "Computation of detonation waves in heterogeneous energetic materials," Journal of Computational Physics 196 (2004) 490-538.

28. Kapila, A., Menikoff, R., Bdzil, J., Son, D., Stewart, D.S., “Two-phase modeling of DDT in granular materials: reduced equations," Physics of Fluids 13 (2001) 3002-3024.

29. Murrone, A., and Guillard, H., "A five equations reduced model for compressible twophase flow problems," Journal of Computational Physics 202 (2005) 664-698. 
30. Kreeft, J.J., and Koren, B., "A new formulation of Kapila's five-equation model for compressible two-fluid flow, and its numerical treatment," Journal of Computational Physics 229 (2010) 6220-6242.

31. Perigaud, G., and Saurel, R., "A compressible flow model with capillary effects," Journal of Computational Physics 209 (2005) 139-178.

32. Cahn, J., and Hilliard, J., "Free energy of nonuniform systems: Interfacial free energy," Journal of Chemical Physics 28 (1958) 258.

33. Petitpas, F., Massoni, J., Saurel, R., Lapebie, E., Munier, L., "Diffuse interface model for high speed cavitating underwater systems," International Journal of Multiphase Flow 35 (2009) 747-759.

34. Chang, C.H., and Ramshaw, J.D., "Dynamical evolution of volume fractions in multipressure multiphase flow models," Physical Review E 77 (2008) 066305:1-7.

35. Braconnier, B., and Nkonga, B., "A all-speed relaxation scheme for interface flows with surface tension," Journal of Computational Physics 228 (2009) 5722-5739. 
(this page intentionally blank) 


\section{Appendix}

\section{Well-Posed, Averaged Conservation Equations for Compressible Multiphase, Multi-Component, and Multi-Material Flows}

\section{Introduction}

Many important "fluid" flows involve a combination of two or more materials having different properties. The multiple phases or components often exhibit relative motion among the phases or material classes. The microscopic motions of the individual constituents are complex and the solution to the micro-level evolutionary equations is difficult. Characteristic of such flows of multi-component materials is an uncertainty in the exact locations of the particular constituents at any particular time. For most practical purposes, it is not possible to exactly predict or measure the evolution of the details of such systems, nor is it even necessary or desirable. Instead, we are usually interested in more gross features of the motion, or the "average" behavior of the system. Here we present descriptive equations that will predict the evolution of this averaged behavior. Due to the complexities of interfaces and resultant discontinuities in fluid properties, as well as from physical scaling issues, it is essential to work with averaged quantities and parameters. We begin by tightening up, or more rigorously defining, our concept of an average. There are several types of averaging. The published literature predominantly contains two types of averaging: volume averaging [Whitaker 1999, Dobran 1991] and time averaging [Ishii 1975]. Occasionally combinations of the two are used. However, we utilize a more general approach by adopting what is known as ensemble averaging.

When the physical system has a large amount of variability, a natural interpretation of the meaning of predictions is in terms of expected values and variances. If there are many different events, or realizations, possible, then the expected value is naturally an "average" over all of these events, or the ensemble of realizations. The ensemble then is the set of all experiments with the same boundary- and initial-conditions, with some properties that we would like to associate with the mean and distribution of the components and their velocities. A realization of the flow is a possible motion that could have happened. Implicit in this concept is the intuitive idea of a "more likely" and a "less likely" realization in the ensemble. Therefore, as we shall see shortly each ensemble of realizations, corresponding to a given physical situation, has a probability measure on subsets of realizations. The ensemble average is the generalization of the elementary idea of adding the values of the variable for each realization, and dividing by the number of observations. The ensemble average then allows the interpretation of phenomena in terms of repeatability of multi-component flows.

One of the nice features of ensemble averaging, as opposed to volume averaging, is that ensemble averaging does not require that a control volume contain a large quantity of a particular component in any given realization. Consider the following example, taken directly from Drew and Lahey (1993), where the average of a particle-fluid mixture is of interest. Gas turbines are eroded by particulate matter suspended in the gas stream passing through the inlet and impacting 
on the various parts of the machine, e.g. the turbine blades. The trajectories of individual particles moving through the gas turbine are very complicated, depending on where and when the particles enter the inlet of the device. Such predictions are, fortunately, seldom required. A prediction, however, that is of interest to the designer is the average, or expected values, of the particle flux (or the concentration and velocities of particles) near parts in the device that are susceptible to erosion. Since the local concentration of particles is proportional to the probability that particles will be at the various points in the device at various times, and the particle velocity field will be the mean velocity that the particles will have if they are at that position in the device, the design engineer will be able to use this information to assess the places where erosion due to particle impact may occur. Notice it may be that there are no times for which there will be many particles in some representative control volume (or representative elementary volume, REV). So, volume averaging, which depends on the concept of having many representative particles in the averaging volume at any instant, will fail. The appropriateness of ensemble averaging is obvious. Here the ensemble is the set of motions of a single particle through the device, given that it started at a random point at the inlet at a random time during the transient flow through the device. Clearly the solution for the average concentration and average velocity gives little information about the behavior of a single particle in the device; however, the information is very appropriate for assessing the probability of damage to the device. Similar examples could be given where time averaging will fail, but where ensemble averaging is again appropriate.

The ensemble average is the more fundamentally based than either time or volume averaging. In fact, both time and volume averaging can be viewed as approximations to the ensemble average, which can be justified, respectively, for steady or homogeneous flow [Drew and Passman 1999].

\section{Ensemble Averaging}

A general method is presented here, based on the ensemble averaging concept [Kashiwa \& Rauenzahn 1994, Lhuillier 1996, Brackbill et.al. 1997, Drew \& Passman 1999], for developing averaged conservation equations for multiple materials, any one of which may be at point $\vec{x}$, at a given instant $t$. With this procedure, the most likely state at a point (the expected value) will be determined simultaneously with which material is most likely to be found at that point. Imagine running an experiment many times and collecting data about the state of the flow at each point $\vec{x}$ and time $t$. This information could include which material or phase is present, material density, velocity, pressure, temperature, concentration, etc. From this information, one can compute the ensemble average. The ensemble average of a generic property $Q_{0}$ of a fluid or material in a process is an average over the realizations

$$
\left\langle Q_{0}\right\rangle(\vec{x}, t)=\frac{1}{N_{R}} \sum_{r=1}^{N_{R}} Q_{0, r}(\vec{x}, t),
$$

where $N_{R}$ is the number of times the process or experiment is repeated, and is a large number. Now imagine that many of the realizations are near duplicates, i.e. they are essentially the same 
state, with $N$ occurrences. We can then rewrite the sum over the realizations as a sum over the number of states $N_{\Gamma}$

$$
\begin{aligned}
\left\langle Q_{0}\right\rangle(\vec{x}, t) & =\frac{1}{N_{R}} \sum_{\Gamma=1}^{N_{\Gamma}} N(\vec{x}, t, \Gamma) Q_{0}(\Gamma) \\
& =\sum_{\Gamma=1}^{N_{\Gamma}} \frac{N(\vec{x}, t, \Gamma)}{N_{R}} Q_{0}(\Gamma) \\
& =\int_{\text {all } \Gamma} Q_{0}(\Gamma) f(\vec{x}, t, \Gamma) d \Gamma,
\end{aligned}
$$

where $f(\vec{x}, t, \Gamma)=\frac{N(\vec{x}, t, \Gamma)}{N_{R}}$ is the probability of the state $\Gamma$ in the ensemble. Note that in the limit of an infinite number of repetitions of the experiment, with a sum over all of the states, we have replaced the summation with an integral form in the definition of the ensemble average. More correctly, because $\int_{\text {all } \Gamma} f(\vec{x}, t, \Gamma) d \Gamma=1.0$, we refer to $f(\vec{x}, t, \Gamma)$ as the probability density.

The state is the full thermodynamic description of the matter at a point $\vec{x}$ and time t. For example,

$$
\Gamma=\left\{\begin{array}{l}
\rho_{0}, \vec{u}_{0}, h_{0}, p_{0}, \tau_{\sim}, \\
\rho_{0}^{1}, \vec{u}_{0}^{1}, h_{0}^{1}, \rho_{0}^{2}, \vec{u}_{0}^{2}, h_{0}^{2}, \cdots, \\
X_{1}, X_{2}, \cdots
\end{array}\right\}
$$

where:

$X_{k}(\vec{x}, t)$ phase or material indicator function : $=1$ if material $k$ is present

$$
=0 \text { otherwise }
$$

$\rho_{0} \quad$ phase or material density

$\vec{u}_{0} \quad$ phase or material velocity

$h_{0} \quad$ phase or material specific enthalpy

$p_{0} \quad$ pressure

$\tau_{\sim} \quad$ deviatoric stress

$\rho_{0}^{s} \quad$ species partial density

$\vec{u}_{0}^{s} \quad$ species velocity

$h_{0}^{s} \quad$ species partial enthalpy,

with 


$$
\begin{aligned}
& \rho_{0}=\sum_{\text {species }} \rho_{0}^{s} \\
& \rho_{0} \vec{u}_{0}=\sum_{\text {species }} \rho_{0}^{s} \vec{u}_{0}^{s} \\
& \rho_{0} h_{0}=\sum_{\text {species }} \rho_{0}^{s} h_{0}^{s} .
\end{aligned}
$$

Other properties may also appear in the above thermodynamic state such as the phase or material temperature $\theta_{0}$, the phase or material specific internal energy $e_{0}$, and the phase or material specific entropy $s_{0}$.

In a typical multiphase flow, the ensemble averages of interest may include

$$
\begin{aligned}
& \text { Material } k \text { volume fraction : } \quad \alpha_{k} \equiv\left\langle X_{k}\right\rangle \\
& \text { Material } k \text { bulk average density : } \quad \hat{\rho}_{k} \equiv\left\langle X_{k} \rho_{0}\right\rangle \\
& \text { Material } k \text { intrinsic average density : } \quad \rho_{k} \equiv \frac{\left\langle X_{k} \rho_{0}\right\rangle}{\alpha_{k}} \\
& \text { Species s in material } k \text { bulk average density : } \quad \hat{\rho}_{k}^{s} \equiv\left\langle X_{k} \rho_{0}^{s}\right\rangle \\
& \text { Species } s \text { in material } k \text { intrinsic average density : } \quad \rho_{k}^{s} \equiv \frac{\left\langle X_{k} \rho_{0}^{s}\right\rangle}{\alpha_{k}} \\
& \text { Material } k \text { velocity : } \quad \vec{u}_{k} \equiv \frac{\left\langle X_{k} \rho_{0} \vec{u}_{0}\right\rangle}{\hat{\rho}_{k}}=\frac{\left\langle X_{k} \rho_{0} \vec{u}_{0}\right\rangle}{\alpha_{k} \rho_{k}} \\
& \text { Material } k \text { total energy : } \quad E_{k} \equiv \frac{\left\langle X_{k} \rho_{0} E_{0}\right\rangle}{\hat{\rho}_{k}}=\frac{\left\langle X_{k} \rho_{0} E_{0}\right\rangle}{\alpha_{k} \rho_{k}} \\
& \text { Material k entropy: } \quad s_{k} \equiv \frac{\left\langle X_{k} \rho_{0} s_{0}\right\rangle}{\hat{\rho}_{k}}=\frac{\left\langle X_{k} \rho_{0} s_{0}\right\rangle}{\alpha_{k} \rho_{k}} \\
& \text { Mean mixture stress : } \quad \underset{\sim}{T} \equiv\langle\underset{\sim}{T}\rangle \\
& \text { Mean } k \text {-material stress : } \quad T_{\sim k} \equiv \frac{\left\langle X_{k} T_{\sim}\right\rangle}{\alpha_{k}} \\
& \text { Pressure (single pressure model) : } \quad p \equiv\left\langle p_{0}\right\rangle \\
& \text { Pressure in } k \text {-material : } \quad p_{k} \equiv \frac{\left\langle X_{k} p_{0}\right\rangle}{\alpha_{k}} \text {. }
\end{aligned}
$$

From a physical viewpoint, the bulk average density of a phase represents a summation of all of the density values that occurred for that phase, divided by the total number of experiments run. The bulk average density corresponds intuitively to the idea of the mass of phase $k$ per unit volume of mixture, or the observed material density. On the other hand, the intrinsic average density physically corresponds to a summation of all of the density values that occurred for that phase, dividing by the number of times in which that phase occurred in the experiments. The 
intrinsic average density corresponds intuitively to the idea of the mass of phase $k$ per unit volume of phase $k$, or the true material density. Some researchers prefer to work with bulk average densities [e.g. Kashiwa and Rauenzahn 1994] while others prefer working with intrinsic densities [e.g. Drew and Passman 1999]. This is mostly an issue of convenience, since one can easily be converted to the other. Here we will use intrinsic averages. Henceforth, when we say average, we shall mean intrinsic average unless indicated otherwise.

For a reasonably broad range of conditions (with common substances), the exact balance equations, valid inside each material, are

$$
\begin{array}{cc}
\dot{\rho}_{0}=-\rho_{0} \nabla \cdot \vec{u}_{0} & \text { Material mass conservation } \\
\dot{\rho}_{0}^{s}=-\rho_{0}^{s} \nabla \cdot \vec{u}_{0}-\nabla \cdot \rho_{0}^{s}\left(\vec{u}_{0}^{s}-\vec{u}_{0}\right)+\dot{r}_{0}^{s} & \text { Species mass conservation } \\
\rho_{0} \dot{\vec{u}}_{0}=\nabla \cdot{\underset{\sim}{0}}_{0}+\rho_{0} \vec{g} & \text { Material momentum balance } \\
\rho_{0} \dot{E}_{0}=\nabla \cdot\left({\underset{\sim}{T}}_{0} \cdot \vec{u}_{0}\right)+\nabla \cdot \vec{q}_{0}+\rho_{0} \vec{g} \cdot \vec{u}_{0}+\rho_{0} \varepsilon_{0} & \text { Material energy conservation } \\
\rho_{0} \dot{s}_{0} \geq \frac{\rho_{0} \varepsilon_{0}}{\theta_{0}}-\nabla \cdot\left(\frac{\vec{q}_{0}}{\theta_{0}}\right) &
\end{array}
$$

For these microscopic balance laws the material derivative has been used, which is defined as

$$
\dot{Q}_{0} \equiv \frac{\partial Q_{0}}{\partial t}+\vec{u}_{0} \cdot \nabla Q_{0} \quad \text { Material derivative }
$$

Let us assume that the total variation of $f$ in the phase space $(\vec{x}, t, \Gamma)$ is [Kashiwa and Rauenzahn 1994]

$$
\frac{\partial f}{\partial t}+\vec{u}_{0} \cdot \nabla f+\dot{\Gamma} \cdot \frac{\partial f}{\partial \Gamma}=\frac{d f}{d t}=0
$$

where we are assuming that as we follow a material point through phase space its probability of occurrence remains constant. Various moments of this equation can be formed, first by multiplying equation (10) by $Q_{0}$, and then averaging this result. It can be shown [Kashiwa and Rauenzahn 1994, here corrected] that the resulting equation is

$$
\frac{\partial}{\partial t}\left\langle Q_{0}\right\rangle+\nabla \cdot\left\langle Q_{0} \vec{u}_{0}\right\rangle=\left\langle\dot{Q}_{0}+Q_{0} \nabla \cdot \vec{u}_{0}\right\rangle
$$

This result is called the moment evolution equation and the details of its derivation are given in the Appendix supplement at the end. The averaged conservation equations are obtained by 
letting our generic $Q_{0}$ be replaced by various "meaningful" functions and then by performing judicious manipulations on the equations to bring about physically useful forms of the equation.

\section{Mass Conservation}

By letting $Q_{0}=X_{k} \rho_{0}$ in equation (11) we get

$$
\begin{aligned}
\frac{\partial\left\langle X_{k} \rho_{0}\right\rangle}{\partial t}+\nabla \cdot\left\langle X_{k} \rho_{0} \vec{u}_{0}\right\rangle & =\left\langle\dot{X}_{k} \rho_{0}+X_{k} \dot{\rho}_{0}+X_{k} \rho_{0} \nabla \cdot \vec{u}_{0}\right\rangle \\
& =\left\langle\dot{X}_{k} \rho_{0}+X_{k}\left(\dot{\rho}_{0}+\rho_{0} \nabla \cdot \vec{u}_{0}\right)\right\rangle
\end{aligned}
$$

Introducing the pure material (microscopic) mass conservation equation and the definition of average results in

$$
\frac{\partial \alpha_{k} \rho_{k}}{\partial t}+\nabla \cdot \alpha_{k} \rho_{k} \vec{u}_{k}=\left\langle\dot{X}_{k} \rho_{0}\right\rangle
$$

Since we are taking time- and spatial-derivatives of functions that are not smooth, this averaged mass conservation equation is to be interpreted in the sense of distributions, or generalized functions [Gelfand and Shilov 1964]. Let us examine the right hand side of this equation in more detail. From the definition of a material derivative we know that

$$
\dot{X}_{k}=\frac{\partial X_{k}}{\partial t}+\vec{u}_{0} \cdot \nabla X_{k}
$$

in a generalized function sense. On the other hand, letting $\vec{u}_{\text {int }}$ denote the velocity of an interface of phase or material $\mathrm{k}$, the material derivative of $X_{k}$ following the interface velocity vanishes

$$
\frac{\partial X_{k}}{\partial t}+\vec{u}_{i n t} \cdot \nabla X_{k}=0
$$

This result can be easily seen by first considering points not on the interface where either $X_{k}=0$ or $X_{k}=1$ and the partial derivatives both vanish, and thus the left side of this equation vanishes identically. For points on the interface, which also move with the interface velocity, the function $X_{k}$ is a jump that remains constant so their material derivatives following the interface vanish. Therefore we can write 


$$
\begin{aligned}
\left\langle\dot{X}_{k} \rho_{0}\right\rangle & =\left\langle\rho_{0}[\left(\frac{\partial X_{k}}{\partial t}+\vec{u}_{0} \cdot \nabla X_{k}\right)-\underbrace{\left(\frac{\partial X_{k}}{\partial t}+\vec{u}_{i n t} \cdot \nabla X_{k}\right)}_{=0}]\right\rangle \\
& =\left\langle\rho_{0}\left(\vec{u}_{0}-\vec{u}_{i n t}\right) \cdot \nabla X_{k}\right\rangle,
\end{aligned}
$$

and the averaged mass conservation equation becomes

$$
\begin{aligned}
\frac{\partial \alpha_{k} \rho_{k}}{\partial t}+\nabla \cdot \alpha_{k} \rho_{k} \vec{u}_{k} & =\left\langle\rho_{0}\left(\vec{u}_{0}-\vec{u}_{i n t}\right) \cdot \nabla X_{k}\right\rangle \\
& \equiv \Omega_{k}^{\text {mass }} .
\end{aligned}
$$

We note that $\nabla X_{k}$ has the sifting property of the Dirac delta function(al). Thus the only contributors are the material interfaces. $\nabla X_{k}$ is aligned with the surface unit normal vector pointing to phase $k$ [Drew 1983, Kataoka and Serizawa 1988]

$$
\nabla X_{k}=\vec{n}_{k} \delta\left(\vec{x}-\vec{x}_{i n t}, t\right)
$$

Thus the $\Omega_{k}^{\text {mass }}$ represents the flux of mass to phase $k$ from the other phases via the interface, usually just referred to as phase change. With no storage of mass at an interface the mass conservation further requires

$$
\sum_{k=1}^{\text {no. of phases }} \Omega_{k}^{\text {mass }}=0
$$

At this point, it is convenient to introduce for later use, the concept of interfacial area density of component $k$. Defined as

$$
A_{k}=-\left\langle\hat{n}_{k} \cdot \nabla X_{k}\right\rangle
$$

where $\hat{n}_{k}$ is the unit external normal to component $k$, it is the expected value of the ratio of the interfacial area (in a small volume) to the (small) volume, in the limit as that volume approaches zero. 


\section{Generic Conservation Equation}

To more expeditiously derive the other conservation equations, let us first derive the averaged balance equation resulting from a generic, microscopic balance equation. Consider the generic, microscopic balance equation

$$
\frac{\partial \rho_{0} \psi_{0}}{\partial t}+\nabla \cdot \rho_{0} \psi_{0} \vec{u}_{0}=\nabla \cdot J_{0}+\rho_{0} g_{0}
$$

or

$$
\frac{d\left(\rho_{0} \psi_{0}\right)}{d t}+\left(\rho_{0} \psi_{0}\right) \nabla \cdot \vec{u}_{0}=\rho_{0} \dot{\psi}_{0}=\nabla \cdot J_{0}+\rho_{0} g_{0}
$$

Equation (16) and (17) hold at each point where sufficient smoothness occurs for the derivatives to be taken, as does its generic jump condition

$$
\llbracket \rho_{0} \psi_{0}\left(\vec{u}_{0}-\vec{u}_{i n t}\right)+J_{0} \rrbracket \cdot \hat{n}=m
$$

where $\psi_{0}$ is the conserved quantity, $J_{0}$ is a molecular or diffusive flux, $g_{0}$ is a source density, and $m$ is the interfacial source of $\psi_{0}$. The symbol $\llbracket \cdot \rrbracket$ here denotes the jump in the enclosed quantity across an interface. Obviously, these quantities must be added to our state space, e.g.

$$
\Gamma=\left\{\begin{array}{l}
\rho_{0}, \vec{u}_{0}, \psi_{0}, J_{0}, \cdots \\
X_{1}, X_{2}, \cdots
\end{array}\right\} .
$$

Let us also define averages of these quantities as

$$
\begin{gathered}
\psi_{k} \equiv \frac{\left\langle X_{k} \rho_{0} \psi_{0}\right\rangle}{\alpha_{k} \rho_{k}} \\
J_{k} \equiv \frac{\left\langle X_{k} J_{0}\right\rangle}{\alpha_{k}} \\
g_{k} \equiv \frac{\left\langle X_{k} \rho_{0} g_{0}\right\rangle}{\alpha_{k} \rho_{k}} .
\end{gathered}
$$

By letting $Q_{0}=X_{k} \rho_{0} \psi_{0}$ in equation (11) we get 


$$
\begin{aligned}
\frac{\partial\left\langle X_{k} \rho_{0} \psi_{0}\right\rangle}{\partial t}+\nabla \cdot\left\langle X_{k} \rho_{0} \psi_{0} \vec{u}_{0}\right\rangle & =\left\langle\frac{d\left(X_{k} \rho_{0} \psi_{0}\right)}{d t}+X_{k} \rho_{0} \psi_{0} \nabla \cdot \vec{u}_{0}\right\rangle \\
& =\left\langle\dot{X}_{k} \rho_{0} \psi_{0}+X_{k} \frac{d\left(\rho_{0} \psi_{0}\right)}{d t}+X_{k} \rho_{0} \psi_{0} \nabla \cdot \vec{u}_{0}\right\rangle \\
& =\left\langle\dot{X}_{k} \rho_{0} \psi_{0}\right\rangle+\left\langle X_{k}\left(\frac{d\left(\rho_{0} \psi_{0}\right)}{d t}+\rho_{0} \psi_{0} \nabla \cdot \vec{u}_{0}\right)\right\rangle \\
& =\left\langle\dot{X}_{k} \rho_{0} \psi_{0}+X_{k} \nabla \cdot J_{0}+X_{k} \rho_{0} g_{0}\right\rangle \\
& =\left\langle\nabla \cdot X_{k} J_{0}\right\rangle-\left\langle J_{0} \cdot \nabla X_{k}\right\rangle+\left\langle\dot{X}_{k} \rho_{0} \psi_{0}\right\rangle+\left\langle X_{k} \rho_{0} g_{0}\right\rangle \\
& =\left\langle\nabla \cdot X_{k} J_{0}\right\rangle-\left\langle J_{0} \cdot \nabla X_{k}\right\rangle+\left\langle\rho_{0} \psi_{0}\left(\vec{u}_{0}-\vec{u}_{i n t}\right) \cdot \nabla X_{k}\right\rangle+\left\langle X_{k} \rho_{0} g_{0}\right\rangle \\
& =\nabla \cdot\left\langle X_{k} J_{0}\right\rangle+\left\langle X_{k} \rho_{0} g_{0}\right\rangle+\left\langle\left[\rho_{0} \psi_{0}\left(\vec{u}_{0}-\vec{u}_{i n t}\right)-J_{0}\right] \cdot \nabla X_{k}\right\rangle .
\end{aligned}
$$

Introducing the fluctuating velocity

$$
\vec{u}_{k}^{\prime}=\vec{u}_{0}-\vec{u}_{k}
$$

into this expression finally results in

$$
\begin{aligned}
\frac{\partial \alpha_{k} \rho_{k} \psi_{k}}{\partial t}+\nabla \cdot \alpha_{k} \rho_{k} \psi_{k} \vec{u}_{k} & =\nabla \cdot \alpha_{k} J_{k}-\nabla \cdot\left\langle X_{k} \rho_{0} \psi_{0} \vec{u}_{k}^{\prime}\right\rangle+\alpha_{k} \rho_{k} g_{k}+\left\langle\left[\rho_{0} \psi_{0}\left(\vec{u}_{0}-\vec{u}_{i n t}\right)-J_{0}\right] \cdot \nabla X_{k}\right\rangle \\
& =\nabla \cdot \alpha_{k} J_{k}-\nabla \cdot\left\langle X_{k} \rho_{0} \psi_{0} \vec{u}_{k}^{\prime}\right\rangle+\alpha_{k} \rho_{k} g_{k}+\left\langle\rho_{0} \psi_{0}\left(\vec{u}_{0}-\vec{u}_{i n t}\right) \cdot \nabla X_{k}\right\rangle-\left\langle J_{0} \cdot \nabla X_{k}\right\rangle \\
& =\nabla \cdot \alpha_{k} J_{k}+\nabla \cdot \alpha_{k} J_{k}^{\text {Fluct }}+\alpha_{k} \rho_{k} g_{k}+\Omega_{k}^{\text {mass }} \psi_{k}^{\text {int }}+\Omega_{k}^{\psi},
\end{aligned}
$$

where $J_{k}^{\text {Fluct }}=-\frac{\left\langle X_{k} \rho_{0} \psi_{0} \vec{u}_{k}^{\prime}\right\rangle}{\alpha_{k}}$ is the flux of $\psi$ due to fluctuations in the phase $k$ velocity, $\psi_{k}^{\text {int }}$ is the effective value of $\psi$ that is transferred to phase $k$ from the other phases due to mass transfer, or phase change, and $\Omega_{k}^{\psi}$ is a flux of $\psi$ to phase $k$ not due to bulk mass transfer from the other phases. This is our generic, averaged balance equation. To obtain balance at the interface, our generic jump balance equation requires the constraint

$$
\sum_{k=1}^{\text {no. of phases }} \Omega_{k}^{\text {mass }} \psi_{k}^{\text {int }}+\Omega_{k}^{\psi}=M
$$

where $M=\langle m\rangle$ is the expected net effect of all the interfacial $\psi$-source terms. 


\section{Species Mass Conservation}

The microscopic species mass balance equation can be written as

$$
\frac{\partial \rho_{0}^{s}}{\partial t}+\nabla \cdot \rho_{0}^{s} \vec{u}_{0}^{s}=\dot{r}^{s}
$$

where $\rho_{0}^{s}$ is the species partial density, $\vec{u}_{0}^{s}$ is the species bulk velocity, and $\dot{r}^{s}$ is the generation or source of the species due to chemical reactions. The species mass balance equation is not usually written this way because we usually don't know much about individual species velocities. Instead, it is usually cast as

$$
\frac{\partial \rho_{0}^{s}}{\partial t}+\nabla \cdot \rho_{0}^{s} \vec{u}_{0}=\nabla \cdot \rho_{0}^{s}\left(\vec{u}_{0}-\vec{u}_{0}^{s}\right)+\dot{r}^{s}
$$

because we have (to a certain extent) acquired empirical knowledge of the behavior of the first term on the right hand side of this equation, as we shall see shortly. Let us now recast this equation as

$$
\frac{\partial}{\partial t}\left(\rho_{0} \frac{\rho_{0}^{s}}{\rho_{0}}\right)+\nabla \cdot\left(\rho_{0} \frac{\rho_{0}^{s}}{\rho_{0}} \vec{u}_{0}\right)=\nabla \cdot\left[\rho_{0} \frac{\rho_{0}^{s}}{\rho_{0}}\left(\vec{u}_{0}-\vec{u}_{0}^{s}\right)\right]+\rho_{0} \frac{\dot{r}^{s}}{\rho_{0}}
$$

which is in the form of our generic, averaged balance equation (20) with the assignments of

$$
\psi_{0}=\frac{\rho_{0}^{s}}{\rho_{0}} \quad J_{0}=\rho_{0} \frac{\rho_{0}^{s}}{\rho_{0}}\left(\vec{u}_{0}-\vec{u}_{0}^{s}\right) \quad g_{0}=\frac{\dot{r}^{s}}{\rho_{0}} .
$$

Thus the averaged species mass balance equation is

$$
\frac{\partial}{\partial t}\left\langle X_{k} \rho_{0}^{s}\right\rangle+\nabla \cdot\left\langle X_{k} \rho_{0}^{s} \vec{u}_{0}\right\rangle=\nabla \cdot\left\langle X_{k} \rho_{0}^{s}\left(\vec{u}_{0}-\vec{u}_{0}^{s}\right)\right\rangle+\left\langle X_{k} \dot{r}^{s}\right\rangle+\left\langle\left[\rho_{0}^{s}\left(\vec{u}_{0}-\vec{u}_{i n t}\right)-\rho_{0}^{s}\left(\vec{u}_{0}-\vec{u}_{0}^{s}\right)\right] \cdot \nabla X_{k}\right\rangle .
$$

Again introducing the fluctuating velocity and the definitions of averaged quantities, our final form of the averaged species mass balance equation is

$$
\begin{aligned}
\frac{\partial \alpha_{k} \rho_{k}^{s}}{\partial t}+\nabla \cdot \alpha_{k} \rho_{k}^{s} \vec{u}_{k} & =\nabla \cdot\left\langle X_{k} \rho_{0}^{s}\left(\vec{u}_{0}-\vec{u}_{0}^{s}\right)\right\rangle & & \text { relative species flux } \\
& -\nabla \cdot\left\langle X_{k} \rho_{0}^{s} \vec{u}_{k}^{\prime}\right\rangle & & \text { fluctuational diffusion } \\
& +\left\langle\rho_{0}^{s}\left(\vec{u}_{0}-\vec{u}_{i n t}\right) \cdot \nabla X_{k}\right\rangle & & \text { phase change } \\
& -\left\langle\rho_{0}^{s}\left(\vec{u}_{0}-\vec{u}_{0}^{s}\right) \cdot \nabla X_{k}\right\rangle & & \text { mass exchange } \\
& +\dot{R}_{k}^{s} & & \text { chemical reactions }
\end{aligned}
$$


where we have defined the average generation rate in phase $k$ due to chemical reactions as $\dot{R}_{k}^{s} \equiv \frac{\left\langle X_{k} \dot{r}^{s}\right\rangle}{\alpha_{k}}$.

\section{Momentum Balance}

The averaged momentum balance equation results from the generic, averaged balance equation (20) with the assignment of

$$
\psi_{0}=\vec{u}_{0} \quad J_{0}={\underset{\sim}{T} 0}_{0} \quad g_{0}
$$

to give

$$
\frac{\partial \alpha_{k} \rho_{k} \vec{u}_{k}}{\partial t}+\nabla \cdot \alpha_{k} \rho_{k} \vec{u}_{k} \vec{u}_{k}=\nabla \cdot \alpha_{k}\left(\underset{\sim k}{T_{k}}+T_{\sim}^{\text {Fluct }}\right)+\alpha_{k} \rho_{k} \vec{g}_{k}+\Omega_{k}^{\text {mom }}+\vec{u}_{k}^{\text {int }} \Omega_{k}^{\text {mass }}
$$

where the fluctuating stress $\underset{\sim}{T}$ Fluct and the interfacial momentum source $\Omega_{k}^{\text {mom }}$ are given by

$$
\underset{\sim k}{T_{k}^{F l u c t}} \equiv-\frac{\left\langle X_{k} \rho_{0} \vec{u}_{k}^{\prime} \vec{u}_{k}^{\prime}\right\rangle}{\alpha_{k}} \quad \Omega_{k}^{m o m} \equiv-\left\langle\underset{\sim 0}{T} \cdot \nabla X_{k}\right\rangle
$$

The averaged interfacial momentum balance constraint (jump condition) is

$$
\sum_{k=1}^{\text {no. of phases }} \Omega_{k}^{\text {mom }}+\vec{u}_{k}^{\text {int }} \Omega_{k}^{\text {mass }}=\vec{M}_{\text {surface tension }},
$$

where $\vec{M}_{\text {surface tension }}$ is the interfacial momentum source, i.e. surface tension source.

\section{Energy Conservation}

The assignment of

$$
\psi_{0}=E_{0}=e_{0}+\frac{1}{2} \vec{u}_{0} \cdot \vec{u}_{0} \quad J_{0}=T_{\sim} \cdot \vec{u}_{0}+\vec{q}_{0} \quad g_{0}=\vec{g}_{0} \cdot \vec{u}_{0}+\varepsilon_{0}
$$

to the variables of the generic, averaged balance equation (20) gives the averaged energy conservation equation 


$$
\begin{aligned}
\frac{\partial}{\partial t} \alpha_{k} \rho_{k}\left(e_{k}+\frac{1}{2} \vec{u}_{k} \cdot \vec{u}_{k}+e_{k}^{F l u c t}\right)+\nabla \cdot \alpha_{k} \rho_{k} \vec{u}_{k}\left(e_{k}+\frac{1}{2} \vec{u}_{k} \cdot \vec{u}_{k}+e_{k}^{\text {Fluct }}\right)= & \nabla \cdot\left[\alpha_{k}\left({\underset{\sim}{k}}_{k}+T_{\sim}^{\text {Fluct }}\right) \cdot \vec{u}_{k}\right] \\
& -\nabla \cdot \alpha_{k}\left(\vec{q}_{k}+\vec{q}_{k}^{\text {Fluct }}\right)+\alpha_{k} \rho_{k}\left(\varepsilon_{k}+\vec{g}_{k} \cdot \vec{u}_{k}\right) \\
& +\Omega_{k}^{\text {energy }}+\Omega_{k}^{\text {mom }} \cdot \vec{u}_{k}^{\text {int }} \\
& +\Omega_{k}^{\text {mass }}\left(e_{k}^{\text {int }}+\frac{1}{2} \vec{u}_{k}^{\text {int }} \cdot \vec{u}_{k}^{\text {int }}\right)
\end{aligned}
$$

where

$$
\begin{aligned}
& e_{k}^{\text {Fluct }} \equiv \frac{1}{2} \frac{\left\langle X_{k} \rho_{0} \vec{u}_{k}^{\prime} \cdot \vec{u}_{k}^{\prime}\right\rangle}{\alpha_{k} \rho_{k}} \quad \text { fluctuation kinetic energy } \\
& \vec{q}_{k}^{F l u c t} \equiv \underbrace{\frac{\left\langle X_{k} \rho_{0} \vec{u}_{k}^{\prime} e_{k}^{\prime}\right\rangle}{\alpha_{k}}}_{\text {fluctution internal energy flux }}+\underbrace{\frac{\left\langle X_{k} T_{\sim 0} \cdot \vec{u}_{k}^{\prime}\right\rangle}{\alpha_{k}}}_{\text {fluctuation shear working }}+\underbrace{\frac{1}{2} \frac{\left\langle X_{k} \rho_{0} \vec{u}_{k}^{\prime}\left(\vec{u}_{k}^{\prime} \cdot \vec{u}_{k}^{\prime}\right)\right\rangle}{\alpha_{k}}}_{\text {fluctuation kinetic energy flux }} \text { fluctuation energy flux } \\
& \varepsilon_{k} \equiv \frac{\left\langle X_{k} \rho_{0} \varepsilon_{0}\right\rangle}{\alpha_{k} \rho_{k}} \quad \text { energy source } \\
& \Omega_{k}^{\text {energy }} \equiv\left\langle\vec{q}_{0} \cdot \nabla X_{k}\right\rangle \quad \text { interfacial heat source } \\
& \Omega_{k}^{m o m} \cdot \vec{u}_{k}^{i n t} \equiv-\left\langle\underset{\sim 0}{T} \cdot \vec{u}_{0} \cdot \nabla X_{k}\right\rangle \quad \text { interfacial work } .
\end{aligned}
$$

The averaged interfacial energy balance constraint (jump condition) is

$$
\sum_{k=1}^{\text {no. of phases }} \Omega_{k}^{\text {energy }}+\Omega_{k}^{\text {mom }} \cdot \vec{u}_{k}^{\text {int }}+\Omega_{k}^{\text {mass }}\left(e_{k}^{\text {int }}+\frac{1}{2} \vec{u}_{k}^{\text {int }} \cdot \vec{u}_{k}^{\text {int }}\right)=\xi \text {, }
$$

where $\xi$ is the interfacial energy source.

The kinetic energy associated with the velocity fluctuations, $e_{k}^{\text {Fluct }}$, is a type of "turbulent" kinetic energy. Sometimes the sum $e_{k}+e_{k}^{\text {Fluct }}$ is interpreted as the effective internal energy per unit mass of phase $k$.

It is sometimes useful to have an expression for the balance of fluctuation kinetic energy, $e_{k}^{\text {Fluct }}$. Its evolutionary description is derived by introducing the partition $\vec{u}_{k}^{\prime}=\vec{u}_{0}-\vec{u}_{k}$ into the microscopic pure phase momentum balance, taking the dot product of this equation with $X_{k} \vec{u}_{k}^{\prime}$, 
then performing the statistical average over configurations (keeping in mind that $\left\langle X_{k} \rho_{0} \vec{u}^{\prime}\right\rangle$ vanishes) to obtain [details are left to the reader, see e.g. Nigmatulin 1990]

$$
\begin{aligned}
\alpha_{k} \rho_{k} \frac{\partial e_{k}^{\text {Fluct }}}{\partial t}+\alpha_{k} \rho_{k} \vec{u}_{k} \cdot \nabla e_{k}^{\text {Fluct }} & =\alpha_{k} T_{\sim k}^{\text {Fluct }}: \nabla \vec{u}_{k} \\
& -\nabla \cdot\left\langle\frac{X_{k} \rho_{0}\left(\vec{u}_{k}^{\prime} \cdot \vec{u}_{k}^{\prime}\right) \vec{u}_{k}^{\prime}}{2}\right\rangle+\left\langle X_{k} \vec{u}_{k}^{\prime} \cdot\left(\nabla \cdot T_{\sim}+\rho_{0} \vec{g}_{0}\right)\right\rangle
\end{aligned}
$$

This equation bares some similarity to the equation of evolution of the fluctuational kinetic energy in a single-phase turbulent fluid [Wilcox 1998]. The first term on the right side describes the influence of the gradient of $\vec{u}_{k}$ on the development of $e_{k}^{\text {Fluct }}$, the second term is expected to diffuse $e_{k}^{\text {Fluct }}$, and the last term represents the power developed by the stresses and external forces [Lhuillier 1996].

For most multiphase flows, including some very (conceptually) simple flows such as gas flow through a packed bed or through a pebble-bed nuclear reactor, the nature of $e_{k}^{\text {Fluct }}$ is somewhat different than that of a turbulent single-phase flow. Contrary to a one-phase fluid in which the fluctuations disappear for slow flows, these fluctuations for a multiphase flow exist however slow the flow. For this reason, $e_{k}^{\text {Fluct }}$ that is produced by hydrodynamic interactions between the phases is sometimes called pseudo-turbulence [Lhuillier 1996].

\section{Entropy Inequality}

The local form of the entropy inequality (8), sometimes called the "Second Law of Thermodynamics," is used to place restrictions on the constitutive relations used to give unique phase or material behaviors. With the assignment of

$$
\psi_{0}=s_{0} \quad J_{0}=-\frac{\vec{q}_{0}}{\theta_{0}} \quad g_{0}=\frac{\varepsilon_{0}}{\theta_{0}}
$$

to the variables of the generic, averaged balance relationship (20) the averaged entropy inequality results,

$$
\frac{\partial \alpha_{k} \rho_{k} s_{k}}{\partial t}+\nabla \cdot \alpha_{k} \rho_{k} s_{k} \vec{u}_{k} \geq \nabla \cdot \alpha_{k}\left(\Phi_{k}+\Phi_{k}^{\text {Fluct }}\right)+\alpha_{k} \rho_{k} S_{k}+\Omega_{k}^{\text {entropy }}+\Omega_{k}^{\text {mass }} s_{k}^{\text {int }}
$$

where

$$
\vec{\Phi}_{k} \equiv-\frac{\left\langle X_{k} \frac{\vec{q}_{0}}{\theta_{0}}\right\rangle}{\alpha_{k}} \quad \text { entropy flux }
$$




$$
\begin{gathered}
\vec{\Phi}_{k}^{\text {Fluct }} \equiv-\frac{\left\langle X_{k} \rho_{0} s_{k}^{\prime} \vec{u}_{k}^{\prime}\right\rangle}{\alpha_{k}} \quad \text { fluctuation entropy flux } \\
S_{k} \equiv \frac{\left\langle X_{k} \frac{\rho_{0} \varepsilon_{0}}{\theta_{0}}\right\rangle}{\alpha_{k} \rho_{k}} \quad \text { entropy source } \\
\Omega_{k}^{\text {entropy }} \equiv\left\langle\frac{\vec{q}_{0}}{\theta_{0}} \cdot \nabla X_{k}\right\rangle \quad \text { interfacial entropy source. }
\end{gathered}
$$

This entropy inequality corresponds to what Drew and Passman (1999) call the microscopic entropy inequality. A macroscopic entropy inequality can be obtained by summing inequalities (30) over all of the phases or materials present in the mixture [Truesdell 1984 and the other authors contained therein]. The macroscopic entropy inequality is useful for placing restrictions on the phasic or material interaction constitutive relations. The averaged interfacial entropy inequality (jump condition) is

$$
\sum_{k=1}^{\text {no. of phases }} \Omega_{k}^{\text {entropy }}+\Omega_{k}^{\text {mass }} s_{k}^{\text {int }} \geq 0
$$

\section{Volume Fraction Propagation Equations}

There remains one very important relationship to derive, a dynamic relationship that effectively reflects boundary conditions at the microscale. It accounts for the fact that the constituent volume fractions can change without affecting the gross motion and, in a sense, models the microstructural force systems operating in the multiphase mixture. Beginning with the previous Lagrangian interface material derivative relationship for $X_{k}$,

$$
\frac{\partial X_{k}}{\partial t}+\vec{u}_{i n t} \cdot \nabla X_{k}=0
$$

this equation is averaged to give

$$
\begin{aligned}
\left\langle\frac{\partial X_{k}}{\partial t}+\vec{u}_{\mathrm{int}} \cdot \nabla X_{k}\right\rangle & =\left\langle\frac{\partial X_{k}}{\partial t}\right\rangle+\left\langle\vec{u}_{\mathrm{int}} \cdot \nabla X_{k}\right\rangle \\
& =\frac{\partial}{\partial t}\left\langle X_{k}\right\rangle+\left\langle\vec{u}_{\mathrm{int}} \cdot \nabla X_{k}\right\rangle \\
& =\frac{\partial \alpha_{k}}{\partial t}+\left\langle\vec{u}_{\mathrm{int}} \cdot \nabla X_{k}\right\rangle=0 .
\end{aligned}
$$


Introducing the fluctuating interface velocity

$$
\vec{u}_{I}^{\prime}=\vec{u}_{i n t}-\vec{u}_{I}
$$

where $\vec{u}_{I}$ is the average interface velocity, into this equation yields

$$
\begin{aligned}
\frac{\partial \alpha_{k}}{\partial t}+\left\langle\vec{u}_{\mathrm{int}} \cdot \nabla X_{k}\right\rangle & =\frac{\partial \alpha_{k}}{\partial t}+\left\langle\left(\vec{u}_{I}+\vec{u}_{I}^{\prime}\right) \cdot \nabla X_{k}\right\rangle \\
& =\frac{\partial \alpha_{k}}{\partial t}+\left\langle\vec{u}_{I} \cdot \nabla X_{k}\right\rangle+\left\langle\vec{u}_{I}^{\prime} \cdot \nabla X_{k}\right\rangle \\
& =\frac{\partial \alpha_{k}}{\partial t}+\vec{u}_{I} \cdot\left\langle\nabla X_{k}\right\rangle+\left\langle\vec{u}_{I}{ }^{\prime} \cdot \nabla X_{k}\right\rangle \\
& =\frac{\partial \alpha_{k}}{\partial t}+\vec{u}_{I} \cdot \nabla \alpha_{k}+\left\langle\vec{u}_{I}^{\prime} \cdot \nabla X_{k}\right\rangle \\
& =\frac{\partial \alpha_{k}}{\partial t}+\vec{u}_{I} \cdot \nabla \alpha_{k}-\Omega_{k}^{v o l}=0
\end{aligned}
$$

where $\Omega_{k}^{v o l}$ (for which a constituitive description is needed) is the driving function for the change of volume fraction $\alpha_{k}$. In summary, this equation is written as

$$
\frac{\partial \alpha_{k}}{\partial t}+\vec{u}_{I} \cdot \nabla \alpha_{k}=\Omega_{k}^{v o l}
$$

Because of the central role that this volume fraction propagation equation plays in modern, wellposed two-phase flow models with correct wave dynamics, we dedicate the next section to its examination.

\section{Seven Equation, Two-Pressure, Two-Velocity Hyperbolic, Well-Posed Models for Two- Phase Flows}

Prior to 1981 there had been attempts to remove the ill-posed nature from the full, two-phase flow equations, e.g. [Ransom and Scofield, 1976], [Stuhmiller, 1977], [Rousseau and Ferch, 1979], [Banerjee and Chan, 1980], [Hancox et.al., 1980], as well as others. All of these researchers were trying to gain closure of this equation system (the classical 6-equations system) through algebraic means - and were meeting with only limited success.

In 1981 [Nguyen, 1981] presented a paper identifying the missing ingredient in compressible two-phase flow. In this paper Nguyen utilized the entropy production for each phase to perform an Onsager-type analysis wherein a bilinear form in the thermodynamic fluxes and their conjugate forces was obtained. From this he arrived at the so-called phenomenological laws, one of which, in first approximation is: 


$$
p_{k}-p_{i}=L_{\alpha k}\left(\frac{\partial \alpha_{k}}{\partial t}+w_{k} \frac{\partial \alpha_{k}}{\partial z}\right)
$$

where the notation is standard, with $w_{k}$ denoting the $z$-component of phase $k$ velocity and $L_{\alpha k}$ denoting a phenomenological coefficient, possibly to be "obtained from the flow structure." To complete the set of closure equations, Nguyen proposed to define the interface pressure $p_{i}$ as

$$
p_{i}=\frac{1}{2} \sum_{k=1}^{2}\left[p_{k}-\alpha_{k} \rho_{k} \xi_{k}\left(w_{1}, w_{2}\right)\right]
$$

where $\xi_{k}$ was to be defined consistently with the physical situation of interest. Nguyen furthermore deemed it reasonable to assume that the phenomenological coefficients were equal or

$$
L_{\alpha 1}=L_{\alpha 2}=L_{\alpha}
$$

With these assumptions Nguyen then obtained a 7-equation model with real characteristics which was hyperbolic and which could be formulated as a well-posed initial-value problem.

However, what Nguyen did not do (at least in that paper) was the following useful manipulation. Adding his equations (49) and (50) to eliminate $p_{i}$ gives

$$
\frac{\partial \alpha}{\partial t}+\frac{w_{1}+w_{2}}{2} \frac{\partial \alpha}{\partial z}=\frac{p_{1}-p_{2}}{2 L_{\alpha}}
$$

As a further enhancement to the equations of Nguyen's paper, let us assume that the phenomenological coefficients are not equal. It is then easily obtained that

$$
\frac{\partial \alpha}{\partial t}+\left(\frac{L_{1} w_{1}+L_{2} w_{2}}{L_{1}+L_{2}}\right) \frac{\partial \alpha}{\partial z}=\frac{p_{1}-p_{2}}{L_{1}+L_{2}}
$$

Now, if $L_{2} \gg L_{1}$ and $w_{1}$ is of the same order as $w_{2}$ then the above relationship is approximately

$$
\frac{\partial \alpha}{\partial t}+w_{2} \frac{\partial \alpha}{\partial z} \approx \frac{p_{1}-p_{2}}{L_{2}} .
$$

These relationships are appealing because now the volume fraction change is governed by a dynamical relationship in which the pressure difference between the two phases drove the phase change. If the phases had the same pressure there would be no change in volume fraction. The denominators on the right hand side, i.e. combinations of the phenomenological coefficients for the two phases would determine how "fast" pressure equilibrium was attained. The phasic advection equation, or volume fraction propagation equation, also exhibited an advection 
velocity that was a weighted combination of phasic velocities (weighted with the phenomenological coefficients).

In 1983, Passman and Nunziato at SNL and Walsh at U. of Florida published a report [Passman, Nunziato, and Walsh 1983], later to become Appendix 5C of Truesdell's classical work on rational thermodynamics [Truesdell, 1984]. In addition to the traditional axioms of balance, an additional balance axiom was elegantly postulated which describes changes in volume fraction. They utilized the idea of workless constraints to describe a method of accounting, in the entropy inequality for the mixture, for the constraint requiring that the mixture be saturated. This resulted in a volume fraction propagation equation, which they called balance of equilibrated force, which accounts for the fact that the constituent volume fractions can change without affecting the gross motion. As they point out, this equation, in a sense, models the microstructural force systems operative in multiphase mixtures.

\{Note: This approach has not seen much acceptance in the two-phase fluid flow community, probably because the equation derivation was postulational [Truesdell, 1984] (as opposed to using some type of averaging) and because of the lack of physical familiarity with the terminology. However, some years later, Dobran at New York University published a monograph [Dobran, 1991] in which rigorous volume averaging is utilized, along with a basic material deformation postulate, to derive additional transport equations for multiphase mixtures that are very similar.\}

Later in 1983 Baer and Nunziato of SNL released a two-phase mixture theory describing the deflagration-to-detonation transition (DDT) in reactive granular materials [Baer and Nuziato, 1983], later published in the open literature as [Baer and Nunziato, 1986]. However, perhaps because of the application context, in addition to its postulational derivation, this method received little attention from the two-phase fluid dynamics community. In this work the entropy inequality for the two-phase mixture was utilized to directly establish a constitutive volume fraction propagation equation, which (in the context of their application) they called the compaction equation (with phase change)

$$
\frac{\partial \alpha_{s}}{\partial t}+v_{s} \frac{\partial \alpha_{s}}{\partial z}=\frac{\alpha_{s} \alpha_{g}}{\mu_{c}}\left[p_{s}-\left(p_{g}+\beta_{s}\right)\right]+\frac{c_{s}^{+}}{\gamma_{s}}
$$

where $\beta_{s}$ is a configuration pressure which resists changes in the packing of the bed or compaction and the coefficient $\mu_{c}$ is a compaction viscosity (again in their application context) which controls the rate at which pressure equilibrium is reached. Though derived in a different manner, this equation can also be viewed as a simpler, special case of Passman et.al.'s balance of equilibrated force equation, mentioned above. This 7-equation compressible two-phase model of Baer and Nunziato was hyperbolic and well-posed. They also constructed apparently successful numerical solution algorithms for this equation set. While the motivating applications for this model are far different from those of the two-phase, nuclear reactor fluid dynamics community, the foundational principles are very similar. Notice the similarities between their volume fraction propagation equation and that of Nguyen over two years earlier. 
Baer and Nunziato's volume fraction evolutionary equation can be more generally stated as

$$
\frac{\partial \alpha_{a}}{\partial t}+v_{a} \frac{\partial \alpha_{a}}{\partial z}=\sum_{j} \frac{\alpha_{a} \alpha_{j}}{\mu_{j, a}}\left[p_{a}-\beta_{a}-\left(p_{j}-\beta_{j}\right)\right]+\frac{c_{a}^{+}}{\gamma_{a}}
$$

where $\beta_{a}$ are configuration pressures and exchange coefficients $\mu_{j, a}$ reflect interactions between the phases. These rate equations, which provide closure of the mixture model, are force balances involving the configurational pressures, phase pressures, and a viscous-like stress associated with rate-dependent volume fraction changes. The relaxation coefficients, $\mu_{j, a}$, have the units of viscosity. Moreover, like Nguyen's (but unlike Passman et.al.'s), these equations are constitutive equations, not field equations. As pointed out in [Baer, 1997], they reflect boundary conditions at the microscale. [Saurel et.al. 1994] recast the volume fraction evolution equation as a microscopic mass density evolution equation. [Kashiwa and Gaffney, 2003] derive a mass density evolution equation having a somewhat different character.

To more intuitively see the need for a dynamic volume fraction evolution equation consider a cell mixture physics model for two-phase flow. Consider a fixed volume $V$ with two immiscible constituents present (e.g. the two constituents may have been advected into a fixed cell volume). They have masses $m_{1}$ and $m_{2}$ occupying volumes $V_{1}$ and $V_{2}$, respectively, such that

$$
V_{1}+V_{2}=V
$$

Each constituent phase has material density $\rho_{1}$ and $\rho_{2}$, so

$$
\begin{aligned}
V & =V_{1}+V_{2} \\
& =\frac{m_{1}}{\rho_{1}}+\frac{m_{2}}{\rho_{2}}
\end{aligned}
$$

or

$$
\begin{aligned}
1 & =\frac{V_{1}}{V}+\frac{V_{2}}{V} \\
& =\alpha_{1}+\alpha_{2} \\
& =\frac{m_{1}}{V \rho_{1}}+\frac{m_{2}}{V \rho_{2}}
\end{aligned}
$$

where $\alpha_{1}=\frac{V_{1}}{V}$ and $\alpha_{2}=\frac{V_{2}}{V}$ are volume fractions of each phase. For each constituent

$$
\rho_{1}=\frac{m_{1}}{V_{1}} \quad \text { and } \quad \rho_{2}=\frac{m_{2}}{V_{2}}
$$

and 


$$
\begin{aligned}
p_{1} & =f_{1}\left(\rho_{1}, I_{1}\right) \\
& =f_{1}\left(\frac{m_{1}}{V_{1}}, I_{1}\right) \\
p_{2} & =f_{2}\left(\rho_{2}, I_{2}\right) \\
& =f_{2}\left(\frac{m_{2}}{V_{2}}, I_{2}\right) .
\end{aligned}
$$

Now $V_{1}$ and $V_{2}$ are adjusted (subject to the $V_{1}+V_{2}=V$ constraint) until the phase pressures are equal to

$$
p=f_{1}\left(\frac{m_{1}}{V_{1}}, I_{1}\right)=f_{2}\left(\frac{m_{2}}{V_{2}}, I_{2}\right)
$$

with the equilibrium (or equilibration) pressure, $p$. At this equilibrium pressure the corresponding phase volumes yield the equilibrium volume fractions

$$
\begin{gathered}
\alpha_{1}^{e}=\frac{V_{1}}{V} \\
\alpha_{2}^{e}=\frac{V_{2}}{V} .
\end{gathered}
$$

This can be accomplished more generally in a dynamical fashion as follows. First, note that

$$
\begin{aligned}
p_{1} & =f_{1}\left(\rho_{1}, I_{1}\right) \\
& =f_{1}\left(\frac{m_{1}}{\alpha_{1} V}, I_{1}\right) \\
p_{2} & =f_{2}\left(\rho_{2}, I_{2}\right) \\
& =f_{2}\left(\frac{m_{2}}{\alpha_{2} V}, I_{2}\right)
\end{aligned}
$$

and also that

$$
\begin{aligned}
\frac{d \alpha_{1}}{d t} & =-\frac{d \alpha_{2}}{d t} \\
\frac{d^{2} \alpha_{1}}{d t^{2}} & =-\frac{d^{2} \alpha_{2}}{d t^{2}} .
\end{aligned}
$$


Intuitively, we now consider the dynamical equation

$$
\frac{d \alpha_{1}}{d t}=\frac{p_{1}-p_{2}}{\tau}
$$

If $\alpha_{1}$ is compressed too much $\left(p_{1}>p_{2}\right)$ then $\alpha_{1}$ will increase with time (relax) letting $p_{1}$ reduce while $\alpha_{2}$ decreases letting $p_{2}$ increase. This process ends when $p_{1}=p_{2}=p$ and thus $\frac{d \alpha_{1}}{d t}=0$. The relaxation rate, $\tau$, controls the rate at which the phases (pressures) equilibrate.

More generally yet, we could even write

$$
\begin{aligned}
(\text { microinertia }) \cdot \frac{d^{2} \alpha_{1}}{d t^{2}}+(\text { compaction viscosity }) \cdot \frac{d \alpha_{1}}{d t} & =(\text { microstructural forces }) \\
& =F
\end{aligned}
$$

The microstructural force $F$ is a relaxation term that is intended to model the driving force or "resistance" exhibited by the mixture to changes in its configuration. For example, if we were compacting a gas-solid particle bed,

$$
F=\left\{\begin{array}{lll}
\alpha_{s} \alpha_{g}\left(p_{s}-p_{g}-\beta_{s}\right) & \text { for } & p_{s}-\beta_{s}>0 \\
-\alpha_{s} \alpha_{g} p_{g} & \text { for } & p_{s}-\beta_{s} \leq 0
\end{array}\right.
$$

in accordance with the view of compaction as an irreversible process. $\beta_{s}$ is the "configuration pressure" of the bed.

If we set the "microinertial" and the "configuration pressure" to zero we are left with

$$
\mu \frac{d \alpha_{1}}{d t}=\alpha_{1} \alpha_{2}\left(p_{1}-p_{2}\right)
$$

or

$$
\frac{d \alpha_{1}}{d t}=\frac{\alpha_{1} \alpha_{2}\left(p_{1}-p_{2}\right)}{\mu}
$$

Note the multiplicative coefficient $\alpha_{1} \alpha_{2}$ in the driving force $F$. This term is included for a couple of reasons: (1) $\alpha_{1} \alpha_{2}$ is roughly proportional to the interfacial area per unit volume, $\frac{A_{i}}{V}$. and (2) better behavior results in the limit of single phase occurring due to disappearance of the other phase, i.e. $\alpha_{1} \rightarrow 0\left(\alpha_{2} \rightarrow 1\right)$ or $\alpha_{2} \rightarrow 0\left(\alpha_{1} \rightarrow 1\right)$. 
In 1998, Saurel and Abgrall [Saurel and Abgrall, 1999], who had used Baer and Nunziato's model with some slight reformulation for similar applications, and who had begun to generalize it and apply it to other multiphase mixtures of interest in a fluid dynamics context, published their 7-equation compressible multifluid/multiphase flow model. Neglecting microinertia, surface tension, and covariance terms (and assuming no mass transfer between phases) their variant is state as,

$$
\begin{gathered}
\frac{\partial \bar{\rho}_{k}}{\partial t}+\nabla \cdot \bar{\rho}_{k} \vec{u}_{k}=0 \\
\frac{\partial \bar{\rho}_{k} \vec{u}_{k}}{\partial t}+\nabla \cdot\left(\bar{\rho}_{k} \vec{u}_{k} \otimes \vec{u}_{k}\right)+\nabla \bar{p}_{k}=p_{I} \nabla \alpha_{k}+\lambda\left(\vec{u}_{m}-\vec{u}_{k}\right) \\
\frac{\partial \bar{\rho}_{k} E_{k}}{\partial t}+\nabla \cdot\left(\bar{\rho}_{k} E_{k} \vec{u}_{k}+\bar{p}_{k} \vec{u}_{k}\right)=p_{I} u_{I} \cdot \nabla \alpha_{k}+\lambda\left(\vec{u}_{m}-\vec{u}_{k}\right) u_{I}+\mu\left(p_{k}-p_{m}\right) p_{I} \\
\frac{\partial \alpha_{k}}{\partial t}+u_{I} \cdot \nabla \alpha_{k}=\mu\left(p_{k}-p_{m}\right)
\end{gathered}
$$

where $\alpha_{k}, \bar{\rho}_{k}=\alpha_{k} \rho_{k}, \vec{u}_{k}, \bar{p}_{k}=\alpha_{k} p_{k}$, and $E_{k}$ represent the fluid phase $k$ "volume fraction", mass density, velocity, pressure, and total energy, respectively. The non-equilibrium two-phase flow model derived in [Saurel et. al., 2003], a variant of the original [Baer and Nunziato, 1986] model, is preferred because of its symmetric formulation. In these models each phase is assumed compressible with its own thermodynamics. The system involves 7 partial differential equations (volume fraction and mass, momentum, and energy balance for each phase) and is hyperbolic. These equations, which represent the balance of mass, momentum, and total energy, and volume fraction evolution, respectively, with specific interphase transfer terms placed on the right-hand sides. In these equations, (for a two-phase flow) $k=1,2$ and correspond, respectively, with $m=2,1 ; p_{I}$ and $u_{I}$ represent the interfacial pressure and velocity. In the Baer-Nunziato model, these variables are chosen as $p_{I}=p_{2}$ and $u_{I}=\vec{u}_{1}$, while the Saurel model utilized the following interfacial values:

$$
u_{I}=\frac{\sum_{k=1,2} \alpha_{k} \rho_{k} u_{k}}{\sum_{k=1,2} \alpha_{k} \rho_{k}} \quad \text { and } \quad p_{I}=\sum_{k=1,2} \alpha_{k} p_{k}
$$

This model contains relaxation parameters $\lambda$ and $\mu$ that determine the rates at which the velocities and pressures of the two phases reach equilibrium. These equations are closed by the saturation constraint for the volume fractions and an equation of state for each fluid, e.g. the stiffened gas equation of state (which holds approximately for a broad range of gases and liquids)

$$
\begin{gathered}
\alpha_{1}+\alpha_{2}=1 \\
p_{k}=\left(\gamma_{k}-1\right) \rho_{k}\left(e_{k}-q_{k}\right)-\gamma_{k} \pi_{k}
\end{gathered}
$$


where $e_{k}$ is the internal energy and $\gamma_{k}, q_{k}$, and $\pi_{k}$ are constants, specific for each phase $k$. Their model is unconditionally hyperbolic and well-posed, and is to be able to solve physical situations for which other models fail. In the original Baer and Nunziato model, which has become more popular in the literature and even has become known as the BN-model, $u_{i}$ is taken equal to the velocity of the less compressible phase and $p_{i}$, the interphase pressure, is taken equal to the pressure of the most compressible phase. In Saurel and Abgrall, $p_{i}$ is taken equal to the mixture pressure and $u_{i}$ to the velocity of the center of mass. In [Lallemand and Saurel, 2000] new and enhanced pressure relaxation procedures are presented for this method.

Generalizations of this theory have appeared in the literature such as the novel Discrete Equation Method (DEM) [Abgrall and Saurel, 2003]. In the traditional approach presented above, the microscopic level, single phase, partial differential equations (PDE's) over an assumed phase topology distribution were first averaged to obtain macroscopic level multiphase equations. Then appropriate simplifying assumptions were made for the macroscopic level PDE's. Finally, though not discussed yet, the macroscopic multiphase flow equations are numerically discretized and the resulting equations solved, using finite difference, finite volume, or finite element methods, to obtain "standard" numerical solutions. With the DEM approach, one first assumes a generic phase distribution topology. Then a discretized solution is developed within the computational cell using, for example, Riemann and approximate Riemann relations. Finally, this partial solution is averaged over the cell volume and time to obtain a meaningful solution. The DEM method carries a pressure and velocity for each phase, and because it effective only solves Euler equations locally, the method is hyperbolic and well-posed, and it gives correct wave dynamic solutions. But it offers an additional bonus; for example, [Chinnayya, Daniel, and Saurel, 2004] use this new homogenization method (DEM) to obtain, not only the seven equation model above, but also explicit closure formulas for $p_{i}$ and $u_{i}$ that are symmetric, compatible with the second law of thermodynamics, and responsible for the fulfillment of interface conditions when dealing with contact/interface problems; they also provide a general explicit formula for $\mu$. Furthermore, in the acoustic, continuous limit this method leads to the equation system (for simplicity with no mass transfer)

$$
\begin{aligned}
& \frac{\partial \alpha_{1}}{\partial t}+\nabla \alpha_{1} \cdot \vec{u}_{I}=\mu\left(p_{1}-p_{2}\right) \\
& \frac{\partial(\alpha \rho)_{1}}{\partial t}+\nabla \cdot(\alpha \rho \vec{u})_{1}=0 \\
& \frac{\partial(\alpha \rho \vec{u})_{1}}{\partial t}+\nabla \cdot(\alpha \rho \vec{u} \otimes \vec{u}+\alpha p I)_{1}=p_{I} \nabla \alpha_{1}+\lambda\left(\vec{u}_{2}-\vec{u}_{1}\right) \\
& \frac{\partial(\alpha \rho E)_{1}}{\partial t}+\nabla \cdot[\alpha(\rho E+p) \vec{u}]_{1}=p_{I} \vec{u}_{I} \cdot \nabla \alpha_{1}+\lambda \vec{u}_{I}^{\prime} \cdot\left(\vec{u}_{2}-\vec{u}_{1}\right)-\mu p_{I}^{\prime}\left(p_{1}-p_{2}\right) \\
& \frac{\partial(\alpha \rho)_{2}}{\partial t}+\nabla \cdot(\alpha \rho \vec{u})_{2}=0 \\
& \frac{\partial(\alpha \rho \vec{u})_{2}}{\partial t}+\nabla \cdot(\alpha \rho \vec{u} \otimes \vec{u}+\alpha p I)_{2}=p_{I} \nabla \alpha_{2}-\lambda\left(\vec{u}_{2}-\vec{u}_{1}\right)
\end{aligned}
$$


$\frac{\partial(\alpha \rho E)_{2}}{\partial t}+\nabla \cdot[\alpha(\rho E+p) \vec{u}]_{2}=p_{I} \vec{u}_{I} \cdot \nabla \alpha_{2}-\lambda \vec{u}_{I}^{\prime} \cdot\left(\vec{u}_{2}-\vec{u}_{1}\right)+\mu p_{I}^{\prime}\left(p_{1}-p_{2}\right)$,

where $\alpha, \rho, \vec{u}, p, E\left(E=e+\frac{1}{2} \vec{u} \cdot \vec{u}\right)$, and $e$ represent the volume fraction, density, velocity, pressure, total energy, and internal energy, respectively.

The interfacial variables have been determined in [Saurel et. al., 2003] to be

$$
\begin{aligned}
& \vec{u}_{I}=\frac{Z_{1} \vec{u}_{1}+Z_{2} \vec{u}_{2}}{Z_{1}+Z_{2}}+\frac{\nabla \alpha_{1}}{\left|\nabla \alpha_{1}\right|} \frac{p_{2}-p_{1}}{Z_{1}+Z_{2}} \\
& p_{I}=\frac{Z_{2} p_{1}+Z_{1} p_{2}}{Z_{1}+Z_{2}}+\frac{Z_{1} Z_{2}}{Z_{1}+Z_{2}} \frac{\nabla \alpha_{1}}{\left|\nabla \alpha_{1}\right|} \cdot\left(\vec{u}_{2}-\vec{u}_{1}\right) .
\end{aligned}
$$

These variables correspond to the interface velocity of, and pressure exerted on, the surface of a two phase control volume, i.e. at locations where volume fraction gradients are present. The average interfacial velocity and pressure acting inside the two-phase control volume are given by

$$
\begin{aligned}
& \vec{u}_{I}^{\prime}=\frac{Z_{1} \vec{u}_{1}+Z_{2} \vec{u}_{2}}{Z_{1}+Z_{2}} \\
& p_{I}^{\prime}=\frac{Z_{2} p_{1}+Z_{1} p_{2}}{Z_{1}+Z_{2}} .
\end{aligned}
$$

Mechanical non-equilibrium is represented with a relaxation process whose rate is controlled by the following parameters:

$\lambda=\frac{1}{2} \mu Z_{1} Z_{2} \quad$ (velocity relaxation rate)

$\mu=\frac{A_{I}}{Z_{1}+Z_{2}} \quad$ (pressure relaxation rate),

where $A_{I}$ represents the specific interfacial area and $Z_{k}$ the acoustic impedance of phase $k$, i.e. $Z_{k}=\rho_{k} c_{k}$.

\section{Reduced or Relaxed Equation Forms}

The single velocity pressure equilibrium model corresponds to the one of [Kapila et al., 2001]. It has been obtained as the asymptotic limit of the [Baer and Nunziato, 1986] model in the limit of both stiff velocity and pressure relaxation. In involves 5 partial differential equations, four conservative and a non-conservative volume fraction equation. The speed of sound resulting from this model corresponds to that of [Wood, 1930] which exhibits a non-monotonic variation with volume fraction. These two difficulties (non-conservativity and non-monotonicity) present serious computational challenges. To circumvent them, [Saurel, Petitpas, and Berry, 2009] 
constructed a pressure non-equilibrium 6-equation model (based on the first reduced model in Kapila et al., 2001) which is also non-conservative, but easier to solve with a relaxation method.

The [Kapila et al., 2001] model is the zero-order approximation of the Baer and Nunziato model with stiff mechanical relaxation. In one dimension (with no mass transfer), with consideration of only two fluids, the model is given as:

$$
\begin{aligned}
& \frac{\partial \alpha_{1}}{\partial t}+u \frac{\partial \alpha_{1}}{\partial x}=\frac{\rho_{2} c_{2}^{2}-\rho_{1} c_{1}^{2}}{\frac{\rho_{1} c_{1}{ }^{2}}{\alpha_{1}}+\frac{\rho_{2} c_{2}{ }^{2}}{\alpha_{2}}} \frac{\partial u}{\partial x} \\
& \frac{\partial(\alpha \rho)_{1}}{\partial t}+\frac{\partial(\alpha \rho)_{1} u}{\partial x}=0 \\
& \frac{\partial(\alpha \rho)_{2}}{\partial t}+\frac{\partial(\alpha \rho)_{2} u}{\partial x}=0 \\
& \frac{\partial \rho u}{\partial t}+\frac{\partial \rho u^{2}+p}{\partial x}=0 \\
& \frac{\partial \rho E}{\partial t}+\frac{\partial(\rho E+p) u}{\partial x}=0
\end{aligned}
$$

where $\alpha, \rho, u, p, E\left(E=e+\frac{1}{2} u^{2}\right)$, and $e$ represent respectively the volume fraction, the mixture density, the velocity, the mixture pressure, the mixture total energy and the mixture internal energy. The mixture internal energy is defined as

$e=Y_{1} e_{1}\left(\rho_{1}, p,\right)+Y_{2} e_{2}\left(\rho_{2}, p\right)$

and the mass fraction is given by: $Y_{k}=\frac{(\alpha \rho)_{k}}{\rho}$.

The mixture density is defined by $\rho=(\alpha \rho)_{1}+(\alpha \rho)_{2}$.

Each fluid is governed by its own convex equation of state (EOS),

$e_{k}=e_{k}\left(\rho_{k}, p\right)$,

which allows the determination of the phases' sound speed,

$c_{k}=c_{k}\left(\rho_{k}, p\right)$.

The mixture pressure $\mathrm{p}$ is determined by solving equation (55), for which, in the particular case of fluids governed by the stiffened gas EOS

$p_{k}=\left(\gamma_{k}-1\right) \rho_{k} e_{k}-\gamma_{k} p_{\infty<k}$,

the resulting mixture EOS reads,

$$
p\left(\rho, e, \alpha_{1}, \alpha_{2}\right)=\frac{\rho e-\left(\frac{\alpha_{1} \gamma_{1} p_{\infty 1}}{\gamma_{1}-1}+\frac{\alpha_{2} \gamma_{2} p_{\infty 2}}{\gamma_{2}-1}\right)}{\frac{\alpha_{1}}{\gamma_{1}-1}+\frac{\alpha_{2}}{\gamma_{2}-1}}
$$


It is straightforward to obtain the entropy equations:

$\frac{d s_{k}}{d t}=0, \quad k=1,2$

Consequently, this model needs specific relations for its closure in the presence of shocks. In the limit of weak shocks, appropriate shock relations have been determined in [Saurel et al., 2007]:

$$
\begin{aligned}
& Y_{k}=Y_{k}^{0}, \\
& \rho(u-\sigma)=\rho^{0}\left(u^{0}-\sigma\right)=m, \\
& p-p^{0}+m^{2}\left(v-v^{0}\right)=0, \\
& e_{k}-e_{k}^{0}+\frac{p+p^{0}}{2}\left(v_{k}^{*}-v_{k}^{0}\right)=0,
\end{aligned}
$$

where $\sigma$ denotes the shock speed and the upperscript ' 0 ' represents the unshocked state.

These relations have been intensively validated against a large experimental data base for weak and strong shocks in the same reference.

Also of interest, [Guillard and Murrone, 2005] use asymptotic analysis, in the limit of zero relaxation time, to reduce the 7-equation two-phase equation model (which contains relaxation terms that drive the system toward pressure and velocity equilibrium) to a five equation reduced hyperbolic system. In [Saurel, Petitpas, and Berry, 2009] a new equation system, relaxed (reduced) from the seven equation model, and numerical method are presented to efficiently perform DNS-like simulations with the diffuse interface method (DIM) approach, and in [Petitpas, et al., 2009] this DIM approach is extended to treat severe flashing with phase change along with an additional non-condensible gaseous phase.

The advantages of this approach is rapidly being recognized. It is important to get the correct wave behavior during transients and mathematical formulations as well as numerical methods must be clean. The methods discussed above provide this behavior and insure well-posedness, for both averaged multiphase flow models and DNS-like models.

\section{Appendix Supplement: Moment Evolution Equation}

It is critical that special attention be given to functional dependencies in deriving the moment evolution equation. Beginning first by defining the ensemble average of some property $Q_{0}$ as

$$
\left\langle Q_{0}\right\rangle(\vec{x}, t) \equiv \int Q_{0}\left(\Gamma_{0}\right) f\left(\vec{x}, t, \Gamma_{0}\right) d \Gamma_{0}
$$


where, for example, $\Gamma_{0}=\left\{\rho_{0}, \vec{u}_{0}, e_{0}, X_{1}, X_{2}, \cdots\right\}$, note that, while $Q_{0}$ depends explicitly upon the state $\Gamma_{0}$, its average value $\left\langle Q_{0}\right\rangle$ depends upon position $\vec{x}$ and time $t$, which it acquired from the probability function $f\left(\vec{x}, t, \Gamma_{0}\right)$. In fact, $\left\langle Q_{0}\right\rangle$ loses its explicit dependence upon $\Gamma_{0}$ by its very definition as an integral over all possible states $\Gamma_{0}$.

The variation of the probability is

$$
d f=\frac{\partial f}{\partial t} d t+\frac{\partial f}{\partial \vec{x}} \cdot d \vec{x}+\frac{\partial f}{\partial \Gamma_{0}} \cdot d \Gamma_{0}
$$

so the time variation of the probability can be written

$$
\frac{d f}{d t}=\frac{\partial f}{\partial t}+\vec{u}_{0} \cdot \nabla f+\dot{\Gamma}_{0} \cdot \frac{\partial f}{\partial \Gamma_{0}}
$$

Multiplying this equation by $Q_{0}\left(\Gamma_{0}\right)$ and integrating over $\Gamma_{0}$-space gives

$$
\int Q_{0} \frac{d f}{d t} d \Gamma_{0}=\int Q_{0} \frac{\partial f}{\partial t} d \Gamma_{0}+\int Q_{0} \vec{u}_{0} \cdot \nabla f d \Gamma_{0}+\int Q_{0} \Gamma_{0} \cdot \frac{\partial f}{\partial \Gamma_{0}} d \Gamma_{0} .
$$

Upon further examination of each term of this expression shows, since $Q_{0}$ does not depend explicitly on time, $t$, that

$$
\begin{aligned}
\int Q_{0}\left(\Gamma_{0}\right) \frac{\partial f}{\partial t} d \Gamma_{0} & =\int \frac{\partial\left(Q_{0} f\right)}{\partial t} d \Gamma_{0} \\
& =\frac{\partial}{\partial t} \int Q_{0} f d \Gamma_{0} \\
& =\frac{\partial}{\partial t}\left\langle Q_{0}\right\rangle(\vec{x}, t) .
\end{aligned}
$$

Similarly, since $Q_{0}$ is independent of $\vec{x}$,

$$
\begin{aligned}
\int Q_{0} \vec{u}_{0} \cdot \nabla f d \Gamma_{0} & =\int \nabla\left(Q_{0} f\right) \cdot \vec{u}_{0} d \Gamma_{0} \\
& =\int \nabla \cdot\left(Q_{0} \vec{u}_{0} f\right) d \Gamma_{0}-\int Q_{0} f\left(\nabla \cdot \vec{u}_{0}\right) d \Gamma_{0} \\
& =\nabla \cdot \int Q_{0} \vec{u}_{0} f d \Gamma_{0}-\int Q_{0}\left(\nabla \cdot \vec{u}_{0}\right) f d \Gamma_{0} \\
& =\nabla \cdot\left\langle Q_{0} \vec{u}_{0}\right\rangle(\vec{x}, t)-\left\langle Q_{0} \nabla \cdot \vec{u}_{0}\right\rangle(\vec{x}, t) .
\end{aligned}
$$

In addition, the $\dot{\Gamma}_{0}$ are independent of $\Gamma_{0}$, so 


$$
\begin{aligned}
\int Q_{0} \dot{\Gamma}_{0} \cdot \frac{\partial f}{\partial \Gamma_{0}} d \Gamma_{0} & =\dot{\Gamma}_{0} \cdot \int Q_{0} \frac{\partial f}{\partial \Gamma_{0}} d \Gamma_{0} \\
& =\dot{\Gamma}_{0} \cdot\left[\int \frac{\partial\left(Q_{0} f\right)}{\partial \Gamma_{0}} d \Gamma_{0}-\int f \frac{\partial Q_{0}}{\partial \Gamma_{0}} d \Gamma_{0}\right] \\
& =\left.\dot{\Gamma}_{0} \cdot Q_{0} f\right|_{\text {limit of } \Gamma_{0}}-\int \dot{\Gamma}_{0} \cdot \frac{\partial Q_{0}}{\partial \Gamma_{0}} f d \Gamma_{0} \\
& =0-\left\langle\dot{\Gamma}_{0} \cdot \frac{\partial Q_{0}}{\partial \Gamma_{0}}\right\rangle \\
& =-\left\langle\frac{\partial Q_{0}}{\partial \Gamma_{0}} \cdot \frac{d \Gamma_{0}}{d t}\right\rangle \\
& =-\left\langle\frac{d Q_{0}}{d t}\right\rangle=-\left\langle\dot{Q}_{0}\right\rangle(\vec{x}, t) .
\end{aligned}
$$

As long as the $Q_{0}$ are physically conserved quantities then

$$
\int Q_{0} \frac{d f}{d t} d \Gamma_{0}=0
$$

Putting all these expressions back into the integral equation above gives the moment evolution equation (11)

$$
\frac{\partial\left\langle Q_{0}\right\rangle}{\partial t}+\nabla \cdot\left\langle Q_{0} \vec{u}_{0}\right\rangle=\left\langle\dot{Q}_{0}+Q_{0} \nabla \cdot \vec{u}_{0}\right\rangle .
$$

II Notice: By comparison with Equation (1.1) of [Kashiwa and Rauenzahn 1994],

$$
\frac{\partial\left\langle Q_{0}\right\rangle}{\partial t}+\nabla \cdot\left\langle Q_{0} \vec{u}_{0}\right\rangle=\left\langle\dot{\Gamma}_{0} \cdot \frac{\partial Q_{0}}{\partial \Gamma_{0}}\right\rangle+\int Q_{0} \frac{D f}{D t} d \Gamma_{0},
$$

theirs should read

$$
\begin{aligned}
\frac{\partial\left\langle Q_{0}\right\rangle}{\partial t}+\nabla \cdot\left\langle Q_{0} \vec{u}_{0}\right\rangle & =\left\langle\dot{\Gamma}_{0} \cdot \frac{\partial Q_{0}}{\partial \Gamma_{0}}\right\rangle+\left\langle Q_{0} \nabla \cdot \vec{u}_{0}\right\rangle+\int Q_{0} \frac{D f}{D t} d \Gamma_{0} \\
& =\left\langle\dot{Q}_{0}+Q_{0} \nabla \cdot \vec{u}_{0}\right\rangle+\int Q_{0} \frac{D f}{D t} d \Gamma_{0}
\end{aligned}
$$

to be correct. \ 


\section{References}

Abgrall, R. and R. Saurel, "Discrete Equations for Physical and Numerical Compressible Multiphase Mixtures," Journal of Computational Physics, 186, 361-396, 2003.

Baer, M.R., "Continuum Mixture Modeling of Reactive Porous Media," Chapter 3 of "HighPressure Shock Compression of Solids IV: Response of Highly Porous Solids to Shock Loading," edited by L. Davison, Y. Horie, and M. Shahinpoor, Springer, New York, 1997.

Baer, M.R. and J.W. Nunziato, "A Theory of Deflagration-to-Detonation Transition (DDT) in Granular Explosives," Sandia National Laboratory report SAND82-0293, Albuquerque, NM, December 1983.

Baer, M.R. and J.W. Nunziato, "A Two-Phase Mixture Theory for the Deflagration-toDetonation Transition (DDT) in Reactive Granular Materials," Int. J. Multiphase Flow, V. 12, p.861-889, 1986.

Banerjee, S. and A.M.C. Chan, "Separated Flow Models I, Analysis of the Averaged and Local Instantaneous Formulations," Int. J. Multiphase Flow, V. 6, p. 1-24, 1980.

Brackbill, J.U., N.L. Johnson, B.A. Kashiwa, and W.B. Vanderheyden, "Multi-phase Flows and Particle Methods," The Fifth Annual Conference of the Computational Fluid Dynamics Society of Canada, Victoria, British Columbia, May 1997.

Chinnayya, Ashwin, Eric Daniel, and Richard Saurel, "Modelling Detonation Waves in Heterogeneous Energetic Materials," Journal of Computational Physics, 196, 490-538, 2004.

Dobran, Flavio, Theory of Structured Multiphase Mixtures, Springer-Verlag, Berlin, 1991.

Drew, D.A., "Mathematical Modeling of Two-Phase Flow," Ann. Rev. Fluid Mech., V. 15, 1983.

Drew, D.A. and Lahey, R.T., "Analytical Modeling of Multiphase Flow," in Particulate TwoPhase Flow, ed. M.C. Roco, Butterworth-Heinemann, Boston, 1993.

Drew, D.A. and Passman, S.L., Theory of Multicomponent Fluids, Springer-Verlag, New York, 1999.

Gelfand, I.M. and Shilov, G.E., Generalized Functions, Vol. I, Academic, New York, 1964.

Guillard, Herve and Angelo Murrone, "A Five Equation Reduced Model for Compressible Two Phase Flow Problems," Journal of Computational Physics, 202, 664-698, 2005. 
Hancox, W.T., R.L. Ferch, W.S. Liu, and R.E. Niemann, "One-Dimensional Models for Transient Gas-Liquid Flows in Ducts," Int. J. Multiphase Flow, V. 6, p. 25-40, 1980.

Ishii, M., Thermo-Fluid Dynamic Theory of Two-Phase Flow, Eyrolles, Paris, 1975.

Kashiwa, B.A. and Rauenzahn, R.M., "A Multimaterial Formalism," Numerical Methods in Multiphase Flows, ASME FED-Vol. 185, p.149-157, 1994.

Kashiwa, Bryan A. and Edward S. Gaffney, Design Basis for CFDLib, Los Alamos National Laboratory report No. LA-UR-03-1295, 2003.

Kataoka, I. and Serizawa, A., "Interfacial Area Concentration and Its Roles in Local Instant Formulation of Two-Phase Flow," in Transient Phenomena in Multiphase Flow, ed. N.H. Afgan, Hemisphere, New York, 1988.

Lallemand, Marie-Helene and Richard Saurel, "Pressure Relaxation Procedures for Multiphase Compressible Flows," INRIA report No. 4038, France, October 2000.

Lhuillier, D., "The Macroscopic Modelling of Multi-Phase Mixtures," in Flow of Particles in Suspensions, ed. U. Schaflinger, Springer-Verlag Wien, New York, 1996.

Nguyen, H. , “One-Dimensional Models for Transient Two-Phase Separated Flow," Third CSNI Specialist Meeting on Transient Two-Phase Flow, California Institute of Technology, Pasadena, California, March 23-25, 1981.

Nigmatulin, R.I., Dynamics of Multiphase Media, Hemisphere, New York, 1990.

Passman, S.L., J.W. Nunziato, and E.K. Walsh, “A Theory of Multiphase Mixtures," Sandia National Laboratory report SAND82-2261, Albuquerque, NM, March 1983.

Petitpas, F., Massoni, J., Saurel, R., Lapebie, E., and Munier, L., "Diffuse interface model for high speed cavitating underwater systems," Journal of Computational Physics, 35, 747-759, 2009.

Ransom, V.H., and M.P. Scofield, "Two-Pressure Hydrodynamic Model for Two-Phase Separated Flow," Idaho National Engineering Laboratory Report SRD-50-76, January 1976 (Revised, February 1976).

Rousseau, J.C. and R.L. Ferch, "A Note on Two-Phase Separated Flow Models," Int. J. Multiphase Flow, V. 5, p. 489-494, 1979.

Saurel, R., A. Forestier, D. Veyret, and J.-C. Loraud, "A Finite Volume Scheme for Two-Phase Compressible Flows,” Int. J. Numerical Methods in Fluids, 18, 803-819 (1994).

Saurel, Richard and Remi Abrgall, "A Multiphase Godunov Method for Compressible Multifluid and Multiphase Flows,” Journal of Computational Physics 150, 425-467, 1999. 
Saurel, R., O. LeMetayer, J. Massoni, and S. Gavrilyuk, "Shock Jump Relations for Multiphase Mixtures with Stiff Mechanical Relaxation,” Shock Waves, 16, 209-232, 2007.

Saurel R., Petitpas, F., and Berry, R.A., "Simple and efficient relaxation methods for interfaces separating compressible fluids, cavitating flows and shocks in multiphase mixtures," Journal of Computational Physics, 228, 1678-1712, 2009.

Stuhmiller, J.H., "The Influence of Interfacial Pressure Forces on the Character of Two-Phase Flow Model Equations," Int. J. Multiphase Flow, V. 3, p. 551-560, 1977.

Truesdell, C., Rational Thermodynamics, $2^{\text {nd }}$ edition, Springer-Verlag, New York, 1984.

Whitaker, S., The Method of Volume Averaging, Kluwer Academic, Dordrecht, 1999.

Wilcox, D.C., Turbulence Modeling for CFD, $2^{\text {nd }}$ edition, DCW Industries, La Canada, CA, 1998. 
(this page intentionally blank) 


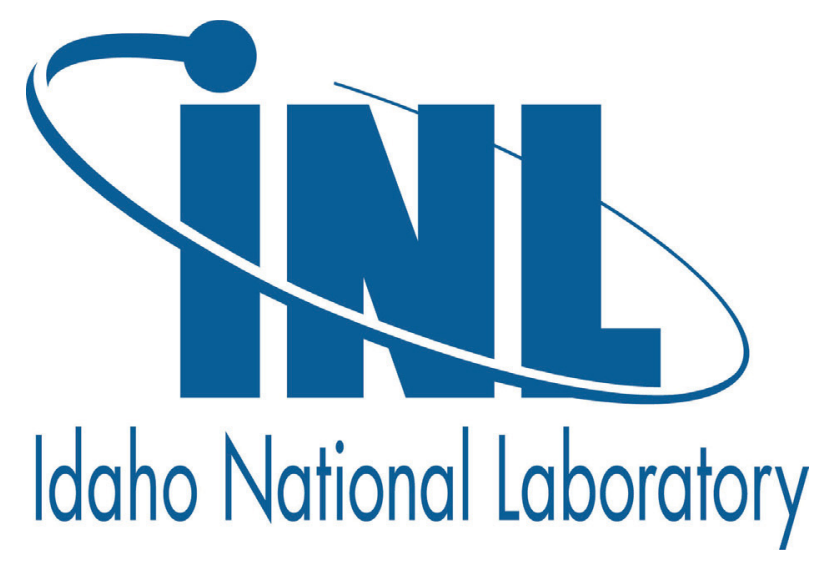

